\title{
A filter-based, mass-conserving lattice Boltzmann method for immiscible multiphase flows
}

\author{
Jianghui Chao ${ }^{1}$, Renwei Mei ${ }^{1, *, \dagger}$, Rajkeshar $\operatorname{Singh}^{2}$ and Wei Shyy ${ }^{3}$ \\ ${ }^{1}$ Department of Mechanical and Aerospace Engineering, University of Florida, Gainesville, FL 32611, U.S.A. \\ ${ }^{2}$ GE Company Global Research, Niskayuna, NY, U.S.A. \\ ${ }^{3}$ Department of Aerospace Engineering, University of Michigan, Ann Arbor, MI 48109, U.S.A.
}

\begin{abstract}
SUMMARY
Some issues of He-Chen-Zhang lattice Boltzmann equation (LBE) method (referred as HCZ model) (J. Comput. Physics 1999; 152:642-663) for immiscible multiphase flows with large density ratio are assessed in this paper. An extended HCZ model with a filter technique and mass correction procedure is proposed based on HCZ's LBE multiphase model. The original HCZ model is capable of maintaining a thin interface but is prone to generating unphysical oscillations in surface tension and index function at moderate values of density ratio. With a filtering technique, the monotonic variation of the index function across the interface is maintained with larger density ratio. Kim's surface tension formulation for diffuseinterface method (J. Comput. Physics 2005; 204:784-804) is then used to remove unphysical oscillation in the surface tension. Furthermore, as the density ratio increases, the effect of velocity divergence term neglected in the original HCZ model causes significant unphysical mass sources near the interface. By keeping the velocity divergence term, the unphysical mass sources near the interface can be removed with large density ratio. The long-time accumulation of the modeling and/or numerical errors in the HCZ model also results in the error of mass conservation of each dispersed phase. A mass correction procedure is devised to improve the performance of the method in this regard. For flows over a stationary and a rising bubble, and capillary waves with density ratio up to 100 , the present approach yields solutions with interface thickness of about five to six lattices and no long-time diffusion, significantly advancing the performance of the LBE method for multiphase flow simulations. Copyright (c) 2010 John Wiley \& Sons, Ltd.
\end{abstract}

Received 21 June 2007; Accepted 26 November 2009

KEY WORDS: lattice Boltzmann method; immiscible multiphase flow; filter; mass conservation

\section{INTRODUCTION}

Multiphase flows often possess large property jumps across interfaces, resulting in multiple time and length scales [1,2]. In addition, the interface shapes and locations are not known a priori, form moving boundaries, and need to be treated as part of the solution. Substantial efforts have been made in the research community in developing techniques for such fluid flow problems. Both continuum (Navier-Stokes) [3-7] and kinetic (such as lattice Boltzmann) [8-10] models have been employed. Overall, the continuum approach has received substantially more attention, including sharp and smooth interface methods [1, 11, 12], Eulerian, Lagrangian, and hybrid approaches $[1,6]$.

${ }^{*}$ Correspondence to: Renwei Mei, 231 MAE-A building, PO Box 116250, University of Florida, Gainesville, FL 32611-6250, U.S.A.

†E-mail: rwmei@ufl.edu

Contract/grant sponsor: NASA Constellation University Institutes Program (CUIP)

Contract/grant sponsor: NASA Glenn Research Center; contract/grant number: NAG3-2930 
The lattice Boltzmann equation (LBE) approach has also been successfully developed to treat numerous fluid flow problems, e.g. porous media flows [13], free surface flows [14], reacting flows [15], and numerous high Reynolds number flows [8]. Because some advantages of the LBE method, such as that the characteristic length scale of the LBE methods is closer to the characteristic length scale of the interfaces of multiphase flows; implementation of the LBE method is relatively simple, the LBE method becomes a prospective candidate for incompressible multiphase flow simulations.

In the context of the LBE method for isothermal immiscible multiphase flow, various interfacial characteristics can be incorporated into the LBE model utilizing a kinetic framework. Employing the diffuse-interface approach [14], the LBE multiphase method does not track the interface explicitly and can handle complex phase topology, including breakup and merger. The phase interface is modeled as a thin zone spanning several lattices over which the fluid/flow properties vary smoothly. The surface tension is modeled as a volumetric force that acts on the fluids over only a thin zone across the interface. The volumetric force is represented in the spatial density gradient (or index function) manner. For immiscible multiphase flows without phase change, this interface modeling in the LBE requires that the interface thickness does not smear off, and dispersed phase mass is conserved, with long-time evolution. The numerical methods used in this modeling should also be numerically stable for large flow/fluid property jumps across the interface.

Some of the popular LBE multiphase models include Shan-Chen (hereinafter referred as SC) inter-particle potential model [10,16], free energy model by Swift et al. [17], He-Shan-Doolen model (hereinafter referred to HSD model) from kinetic theory of dense fluid [18], and an extension by He-Chen-Zhang (the HCZ model) [9]. In the SC model, the interface is modeled through nonlocal fluid particle interaction. It is incorporated into the LBE through an additional forcing term added to the macroscopic velocity $[10,16]$. The SC model has been successfully applied in some multiphase flow simulations, including stationary droplet [10], oscillation of a capillary wave [16], and drag and virtual mass forces in bubbly suspensions [19]. However, in this model, temperature is not consistent with the thermodynamics definition, the surface tension coefficient cannot be freely chosen according to the fluid property, and the viscosities of all phases must be the same. The free energy model of Swift et al. [17] does not suffer from such limitations as in the SC model. It has been successfully used to simulate some multiphase flows, such as stationary bubble/droplet, capillary wave, and phase separation in a narrow capillary [17], two-dimensional bubble in Poiseuille flow [20]. However, the Galilean invariance cannot be maintained in this model [21]. In the HSD model the kinetic theory of dense gases is applied to model phase segregation and surface tension. It overcomes the limitations of the SC model while maintaining the Galilean invariance. The major drawback of the HSD model is its numerical instability for flows with large gradients arising from interfacial forcing terms. This drawback of the HSD model, shared by all LBE multiphase models, has not been adequately addressed in the literature. In the HCZ model, introducing a second LBE alleviates the numerical instability. In this second LBE the large gradient term is multiplied by a term that is proportional to the Mach number. The first LBE is used to track interfaces and its function is similar to that of a fixed grid (Eulerian) method, such as the level set method and the volume-of-fluid method. With the improved numerical stability, the Rayleigh-Taylor instability (RTI) with density ratio up to about 20 [9] and the two-phase Rayleigh-Benard convection with a deformable interface [22] have been successfully simulated using the HCZ model. The computational results also show that the HCZ model is comparable in accuracy with the macroscopic CFD method [21]. The detailed assessments for these three major LBE multiphase models can be found in Nourgaliev et al. work [21].

Recently these LBE multiphase models have been extended to flows with large density ratios. Inamuro et al. [23] used a free energy model with a projection method for pressure correction to simulate multiphase flows with large density ratio. Zheng et al. [24] also used a free energy model, and two LBEs for flows with large density ratio, like those in the HCZ model, to capture interface and represent momentum evolution. Lee and Lin model [14] for multiphase flows with large density ratio differs only slightly from the HCZ model. Instead of using index function, Lee and Lin directly used density as the macroscopic variable calculated from the interface-capturing 
LBE. They also used a potential form for surface tension formulation and hybrid discretizations for the forcing terms.

While these models can handle larger density ratios between phases, their capability of maintaining non-diffuse interface thickness for long-time evolution, as in the HCZ model (See Figure 5 in [9]), has not been adequately examined. If the interface diffuses in time, the interfacial forcing terms associated with spatial density (or index function) gradients, and hence the interfacial physics being modeled, will change accordingly. The non-diffuse interface thickness is thus very important for interfacial dynamics simulation using the LBE method.

Another critical factor for immiscible multiphase flows is mass conservation. In the LBE multiphase models, due to numerical modeling and/or numerical errors, a dispersed phase volume may change with time although its density remains the same, leading to incorrect mass and momentum distributions. This problem is worsened as the density ratio increases because the numerical error due to the forcing term calculations becomes larger. To date, this issue has not been investigated in the literature.

In this paper the HCZ model is adopted as the test model because of its capability of maintaining non-diffuse interface thickness for long-time evolution. Some new issues of the HCZ model are assessed for density ratio up to 100 , such as compressibility effect and mass conservation. Several new treatments, which are used to overcome the new difficulties of the HCZ model for large density ratios, are also assessed in this paper. A nonlinear filter technique [25] and a new surface tension formulation from diffuse-interface method [26] are used to remove the unphysical oscillations caused by the surface tension treatment. The interfacial compressibility effect, which was neglected in the original HCZ model, can lead to unphysical mass sources/sinks near-interface regions when the density ratio is large. This aspect is considered in our approach. Furthermore, a correction step is introduced to keep the mass of the dispersed phase conserved.

In the following, the numerical methods associated with the HCZ model, including the interfacial compressibility effect, the surface tension formulation, the filter technique for index function, and the mass correction for the dispersed phase are presented in Section 2. The performance of the improved LBE technique is assessed in Section 3 by simulating flows around a stationary and a rising bubble, and capillary waves. We end the paper with a summary and conclusion.

\section{THE LBE METHOD AND THE PROPOSED IMPROVEMENT}

\subsection{The HCZ model with interfacial compressibility effect}

In the HCZ multiphase model [9] two LBEs are used to describe the evolutions of index function and pressure. The index function is used to track interfaces between different phases. These two LBEs were derived from two discretized Boltzmann equations:

$$
\begin{gathered}
\frac{\partial f_{\alpha}}{\partial t}+e_{\alpha} \cdot \nabla f_{\alpha}=-\frac{f_{\alpha}-f_{\alpha}^{\mathrm{eq}}}{\tau}+\frac{\left(e_{\alpha}-\boldsymbol{u}\right) \cdot \nabla \psi(\phi)}{\phi R T} f_{\alpha}^{\mathrm{eq}} \\
\frac{\partial g_{\alpha}}{\partial t}+\mathbf{e}_{\alpha} \cdot \nabla g_{\alpha}=-\frac{g_{\alpha}-g_{\alpha}^{\mathrm{eq}}}{\tau}+\left(\mathbf{e}_{\alpha}-\mathbf{u}\right) \cdot\left[\Gamma_{\alpha}(\mathbf{u})\left(\mathbf{F}_{\mathrm{s}}+\mathbf{G}\right)-\left(\Gamma_{\alpha}(\mathbf{u})-\Gamma_{\alpha}(\mathbf{0})\right) \nabla \psi(\rho)\right]
\end{gathered}
$$

where $\mathbf{F}_{s}$ and $\mathbf{G}$ are surface force and gravity, respectively. The hydrodynamic properties can be obtained from the distribution functions $f$ and $g$

$$
\phi=\sum_{\alpha} f_{\alpha}, \quad p_{\mathrm{h}}=\sum_{\alpha} g_{\alpha}, \quad \rho R T \boldsymbol{u}=\sum_{\alpha} e_{\alpha} g_{\alpha}
$$

where $\phi$ is index function, $p_{\mathrm{h}}$ is hydrodynamic pressure, $\boldsymbol{u}$ is macroscopic velocity, $T$ is temperature, and $R$ is gas constant. In Equation (1), $f_{\alpha}$ denotes $f\left(\boldsymbol{x}, \boldsymbol{e}_{\alpha}, t\right)$, which is the distribution function in the direction of the $\alpha$ th discrete velocity $\boldsymbol{e}_{\alpha}, \tau$ is dimensionless relaxation time, and $\boldsymbol{x}$ represents 
physical space coordinate, $f_{\alpha}^{\mathrm{eq}}$ is the corresponding equilibrium distribution function in the discrete velocity space

$$
f_{\alpha}^{\mathrm{eq}}=\phi \Gamma(\boldsymbol{u})
$$

where $\phi$ is the index function and

$$
\Gamma(\boldsymbol{u})=\frac{1}{(2 \pi R T)^{D / 2}} \exp \left[-\frac{(\boldsymbol{\xi}-\boldsymbol{u})^{2}}{2 R T}\right]
$$

In Equation (2), the distribution function $g$ is defined from the distribution function $f$ by

$$
g_{\alpha}=R T f_{\alpha}+\Gamma(0) \psi(\rho)
$$

The corresponding equilibrium distribution function $g_{\alpha}^{\mathrm{eq}}$ is

$$
g_{\alpha}^{\mathrm{eq}}=R T f_{\alpha}^{\mathrm{eq}}+\Gamma(0) \psi(\rho)
$$

The functions $\psi$ in Equations (1), (2), and (5) represent exclusively the volumetric effect, and are defined by

$$
\begin{gathered}
\psi(\rho)=p_{\mathrm{h}}-\rho R T \\
\psi(\phi)=p_{\text {th }}-\phi R T
\end{gathered}
$$

where $p_{\text {th }}$ is thermodynamic pressure calculated from equation of state. He et al. [9] used CarnahanStarling equation of state in their model $[27,28]$

$$
p_{\text {th }}=\phi R T \frac{1+\frac{b \phi}{4}+\left(\frac{b \phi}{4}\right)^{2}-\left(\frac{b \phi}{4}\right)^{3}}{\left(1-\frac{b \phi}{4}\right)^{3}}-a \phi^{2}
$$

where $a$ and $b$ are two constants. From the van der Waals theory, if fluid temperature is lower than its critical point, phase segregation appears due to the molecular attraction. In the $p-V-T$ state diagram, the curve plotted from Equation (9) has a mechanical unstable portion, which represents the different separated phases [9]. Therefore, Equation (9) plays a key role in phase segregation in the HCZ model.

Generally the gradient of $\psi(\rho)$ in Equation (2) can be very large across interfaces because of the sharp change of phase densities. By introducing another distribution function $g$, the effect of the gradient of $\psi(\rho)$ is alleviated to some extent by multiplying $\Gamma(\boldsymbol{u})-\Gamma(\boldsymbol{\theta})$, which is proportional to the Mach number under the limit of incompressibility.

In the HCZ model, the following incompressibility assumption is used when deriving Equation (2) (See He et al. paper for details [9])

$$
\frac{\mathrm{D} \psi}{\mathrm{D} t}=\frac{\partial \psi}{\partial t}+\xi \cdot \nabla \psi=\frac{\partial \psi}{\partial t}+\boldsymbol{u} \cdot \nabla \psi+\xi \cdot \nabla \psi-\boldsymbol{u} \cdot \nabla \psi=\frac{\mathrm{d} \psi}{\mathrm{d} t}+(\xi-\boldsymbol{u}) \cdot \nabla \psi
$$

in which

$$
\frac{\mathrm{d} \psi}{\mathrm{d} t}=\frac{\mathrm{d} \psi}{\mathrm{d} \rho} \frac{\mathrm{d} \rho}{\mathrm{d} t}=\frac{\mathrm{d} \psi}{\mathrm{d} \rho}\left(\frac{\partial \rho}{\partial t}+\boldsymbol{u} \cdot \nabla \rho\right)=0
$$

was used by assuming $\boldsymbol{\nabla} \cdot \boldsymbol{u}=0$. Although $\boldsymbol{\nabla} \cdot \boldsymbol{u}=0$ is correct theoretically for individual phases, this condition is not exactly satisfied across interfaces during computations [29]. The effect of $\boldsymbol{\nabla} \cdot \boldsymbol{u}$ which represents the compressibility effect across interfaces therefore should remain in the LBE model. Following He et al. [9] that

$$
\frac{\mathrm{d} \psi}{\mathrm{d} t}=\frac{\mathrm{d} \psi}{\mathrm{d} \rho} \frac{\mathrm{d} \rho}{\mathrm{d} t}
$$


we have

$$
\frac{\mathrm{d} \psi}{\mathrm{d} t}=\frac{\mathrm{d} \psi}{\mathrm{d} \rho} \frac{\mathrm{d} \rho}{\mathrm{d} t}=\frac{\mathrm{d} \psi}{\mathrm{d} \rho}\left(\frac{\partial \rho}{\partial t}+\boldsymbol{u} \cdot \nabla \rho\right)=\frac{\mathrm{d} \psi}{\mathrm{d} \rho}\left(\frac{\partial \rho}{\partial t}+\nabla \cdot(\rho \boldsymbol{u})-\rho \nabla \cdot \boldsymbol{u}\right)
$$

By taking $\partial \rho / \partial t+\nabla \cdot(\rho \boldsymbol{u})=0$, Equation (11) becomes

$$
\frac{\mathrm{d} \psi}{\mathrm{d} t}=-\frac{\mathrm{d} \psi}{\mathrm{d} \rho}(\rho \nabla \cdot \boldsymbol{u})
$$

Substituting Equation (12) into Equation (10), the derivative $D \psi / D t$ becomes

$$
\frac{\mathrm{D} \psi}{\mathrm{D} t}=-\frac{\mathrm{d} \psi}{\mathrm{d} \rho}(\rho \nabla \cdot \boldsymbol{u})+(\xi-u) \cdot \nabla \psi
$$

With this additional term, the LBE for the distribution function $g$ can be written as

$$
\frac{\partial g_{\alpha}}{\partial t}+\boldsymbol{e}_{\alpha} \cdot \nabla g_{\alpha}=-\frac{g_{\alpha}-g_{\alpha}^{\mathrm{eq}}}{\tau}+\left(\boldsymbol{e}_{\alpha}-\boldsymbol{u}\right) \cdot\left[\Gamma_{\alpha}(\boldsymbol{u})\left(\boldsymbol{F}_{\mathrm{s}}+\boldsymbol{G}\right)-\left(\Gamma_{\alpha}(u)-\Gamma_{\alpha}(0)\right) \nabla \psi\right]-\Gamma_{\alpha}(0) \rho \frac{\mathrm{d} \psi}{\mathrm{d} \rho} \nabla \cdot \boldsymbol{u}
$$

In Equation (14), the surface tension in the $\mathrm{HCZ}$ model is given as

$$
\boldsymbol{F}_{\mathrm{S}}=\kappa \rho \nabla \nabla^{2} \rho
$$

The above formulation is equivalent to the following macroscopic equations

$$
\begin{gathered}
\frac{\partial \phi}{\partial t}+\nabla \cdot(\phi \boldsymbol{u})=-\lambda \nabla \cdot\left[\frac{\phi}{\rho} \nabla p(\rho)-\nabla p(\phi)\right] \\
\frac{\partial \rho}{\partial t}+\nabla \cdot(\rho \boldsymbol{u})=0 \\
\rho\left[\frac{\partial \boldsymbol{u}}{\partial t}+(\boldsymbol{u} \cdot \nabla) \boldsymbol{u}\right]=-\nabla p+\nabla \cdot[\rho v(\nabla \boldsymbol{u}+\boldsymbol{u} \nabla)]+\kappa \rho \nabla \nabla^{2} \rho+\boldsymbol{G}
\end{gathered}
$$

The main difference in the formulation between the present work and that of HCZ is in the retention of the RHS term in Equation (12) and the last RHS term in Equation (14). Although the continuity equation from the $\mathrm{HCZ}$ model $((1 / \rho R T) \partial p / \partial t+\nabla \cdot \boldsymbol{u}=0)$ is theoretically equivalent to our present continuity equation (Equation (17)), they have different performances in terms of numerical errors, especially for multiphase flows with interfaces [29]. For completeness, the derivation of the continuity equation $\partial \rho / \partial t+\nabla \cdot(\rho \boldsymbol{u})=0$ is given in the Appendix. The derivation of the momentum equation is exactly the same as that of the HCZ model $[9,30]$. It is not given in this paper for brevity.

To obtain second-order accuracy and maintain the explicit computational scheme, He et al. [9] further introduced two new variables, which are

$$
\begin{gathered}
\bar{f}_{\alpha}=f_{\alpha}+\frac{\left(\boldsymbol{e}_{\alpha}-\boldsymbol{u}\right) \cdot \nabla \psi(\phi)}{2 R T} \Gamma_{\alpha}(\boldsymbol{u}) \delta_{t} \\
\bar{g}_{\alpha}=g_{\alpha}-\frac{1}{2}\left(\boldsymbol{e}_{\alpha}-\boldsymbol{u}\right)\left[\Gamma_{\alpha}(\boldsymbol{u})\left(\boldsymbol{F}_{s}+\boldsymbol{G}\right)-\left(\Gamma_{\alpha}(\boldsymbol{u})-\Gamma_{\alpha}(\mathbf{0})\right) \nabla \psi(\rho)\right] \delta_{t}
\end{gathered}
$$

Including the last term in Equation (14), the new variable $\bar{g}_{\alpha}$ can be rewritten as

$$
\bar{g}_{\alpha}=g_{\alpha}-\frac{1}{2}\left(\boldsymbol{e}_{\alpha}-\boldsymbol{u}\right)\left[\Gamma_{\alpha}(u)\left(\boldsymbol{F}_{s}+\boldsymbol{G}\right)-\left(\Gamma_{\alpha}(u)-\Gamma_{\alpha}(0)\right) \nabla \psi\right] \delta_{t}-\frac{1}{2} \Gamma_{\alpha}(0) \frac{\mathrm{d} \psi}{\mathrm{d} \rho} \nabla \cdot \boldsymbol{u}
$$


The LBEs in terms of these two new variables are

$$
\begin{aligned}
\bar{f}_{\alpha}\left(\boldsymbol{x}+\boldsymbol{e}_{\alpha} \delta_{t}, t+\delta_{t}\right)-\bar{f}_{\alpha}(\boldsymbol{x}, t)= & -\frac{\bar{f}_{\alpha}(\boldsymbol{x}, t)-f_{\alpha}^{\mathrm{eq}}(\boldsymbol{x}, t)}{\tau}-\frac{2 \tau-1}{2 \tau} \frac{\left(\boldsymbol{e}_{\alpha}-\boldsymbol{u}\right) \cdot \nabla \psi(\phi)}{R T} \Gamma_{\alpha}(\boldsymbol{u}) \delta_{t} \\
\bar{g}_{\alpha}\left(\boldsymbol{x}+\boldsymbol{e}_{\alpha} \delta_{t}, t+\delta_{t}\right)-\bar{g}_{\alpha}(\boldsymbol{x}, t)= & -\frac{g_{\alpha}-g_{\alpha}^{\mathrm{eq}}}{\tau}+\left(\boldsymbol{e}_{\alpha}-\boldsymbol{u}\right) \cdot\left[\Gamma_{\alpha}(\boldsymbol{u})\left(\boldsymbol{F}_{\mathrm{s}}+\boldsymbol{G}\right)-\left(\Gamma_{\alpha}(u)-\Gamma_{\alpha}(0)\right) \nabla \psi\right] \delta_{t} \\
& -\Gamma_{\alpha}(0) \rho \frac{\mathrm{d} \psi}{\mathrm{d} \rho} \nabla \cdot \boldsymbol{u} \delta_{t}
\end{aligned}
$$

The macroscopic variables can be calculated from the moments of the new distribution functions

$$
\begin{gathered}
\phi=\sum \bar{f}_{\alpha}, \\
p_{\mathrm{h}}=\sum \bar{g}_{\alpha}-\frac{1}{2} \boldsymbol{u} \cdot \nabla \psi(\rho) \delta_{t} \\
\rho R T \boldsymbol{u}=\sum e_{\alpha} \bar{g}_{\alpha}+\frac{1}{2} R T\left(\boldsymbol{F}_{\mathrm{s}}+\boldsymbol{G}\right) \delta_{t}
\end{gathered}
$$

Density and viscosity can be computed through a linear interpolation from $\phi$

$$
\begin{gathered}
\rho=\rho_{1}+\frac{\phi-\phi_{1}}{\phi_{\mathrm{h}}-\phi_{1}}\left(\rho_{\mathrm{h}}-\rho_{1}\right) \\
v=v_{1}+\frac{\phi-\phi_{1}}{\phi_{\mathrm{h}}-\phi_{1}}\left(v_{\mathrm{h}}-v_{1}\right)
\end{gathered}
$$

For D2Q9 lattice Boltzmann model, the function $\Gamma(\boldsymbol{u})$ in Equation (4) is

$$
\Gamma_{\alpha}(\boldsymbol{u})=w_{\alpha}\left[1+\frac{3 \boldsymbol{e}_{\alpha} \cdot \boldsymbol{u}}{c^{2}}+\frac{9\left(\boldsymbol{e}_{\alpha} \cdot \boldsymbol{u}\right)^{2}}{c^{2}}-\frac{3 \boldsymbol{u}^{2}}{2 c^{2}}\right]
$$

where $c=\delta x / \delta t, \delta x$ and $\delta t$ are lattice units in length and time, respectively; $w_{\alpha}$ is weighted coefficient given by

$$
w_{0}=\frac{4}{9}, \quad w_{1}=w_{3}=w_{5}=w_{7}=\frac{1}{9}, \quad w_{2}=w_{4}=w_{6}=w_{8}=\frac{1}{36},
$$

and the discrete velocities $\left(\boldsymbol{e}_{\alpha}\right)$ are as follows:

$$
\begin{aligned}
& e_{0}=0 \\
& e_{\alpha}=c[\cos ((\alpha-1) \pi / 4), \sin ((\alpha-1) \pi / 4)] \quad \text { for } \alpha=1,3,5,7 \\
& e_{\alpha}=\sqrt{2} c[\cos ((\alpha-1) \pi / 4), \sin ((\alpha-1) \pi / 4)] \quad \text { for } \alpha=2,4,6,8
\end{aligned}
$$

The kinematic viscosity is independent of the surface force and is related to the non-dimensional relaxation time as

$$
v=(\tau-0.5) R T \delta_{t}
$$

Zhang et al. [30] have used the following integral relationship to analytically relate surface tension $\sigma$ with the coefficient $\kappa$ :

$$
\sigma=\kappa I(a)=\kappa \int_{-\infty}^{\infty}\left(\frac{\partial \phi}{\partial z}\right)^{2} \mathrm{~d} z
$$

where $z$ is a direction normal to the interface.

In Equation (23), the term $[\Gamma(u)-\Gamma(0)] \nabla \psi$ can be written as

$$
\left[\Gamma_{\alpha}(u)-\Gamma_{\alpha}(0)\right] \nabla \psi=w_{\alpha}\left[\frac{3 \boldsymbol{e}_{\alpha} \cdot \boldsymbol{u}}{c^{2}}+O\left(\boldsymbol{u}^{2}\right)\right] \nabla \psi
$$


As the high-order term $O\left(\boldsymbol{u}^{2}\right)$ is proportional to the square of the Mach number, they can be neglected under the low-Mach number condition.

The last term in Equation (23) needs further modification because $\mathrm{d} \psi / \mathrm{d} \rho$ is not easily obtained from computations. From the definition $\psi=p_{\mathrm{h}}-\rho R T$, we have

$$
\frac{\mathrm{d} \psi}{\mathrm{d} \rho}=\frac{\mathrm{d} p_{\mathrm{h}}}{\mathrm{d} \rho}-R T .
$$

Away from the interfaces, hydrodynamic pressure and thermodynamic pressure are equivalent, yielding $\mathrm{d} p_{\mathrm{h}} / \mathrm{d} \rho-R T=0$. The RHS of Equation (35) vanishes, so does the last term in Equation (23). Around the interfaces, the pressure gradient may be mainly balanced by the inertial force and surface tension. As the density gradient around the interfaces is of order $O(1)$, and the pressure gradient may be much smaller than the density gradient if surface tension is small enough and low-Mach number limit is still valid $\left(\mathrm{d} p_{\mathrm{h}} \sim O\left(M a^{2}\right)\right)$. With these assumptions, we can have

$$
\frac{\mathrm{d} p_{\mathrm{h}}}{\mathrm{d} \rho} \ll R T
$$

around the interfaces. The last term in Equation (23) can be rewritten as

$$
-\frac{\mathrm{d} \psi}{\mathrm{d} \rho} \rho \nabla \cdot \boldsymbol{u} \simeq \rho R T \nabla \cdot \boldsymbol{u}
$$

Note that Equation (37) is valid not only around the interface, but also away from the interface where $\boldsymbol{\nabla} \cdot \boldsymbol{u} \sim 0$ is maintained. To keep the above assumptions valid, any factors making large local surface tension, like very large interface curvature, should be avoided. This is one limitation of the present model. However, this limitation is not restrictive only to our model; it is a common numerical limitation for many numerical methods for incompressible two-phase flows. Special treatments should be adopted to artificially remove the large local interface curvature effect due to large interface deformation [31].

\subsection{Surface force formulation}

The surface tension in the HCZ model is calculated from Equation (18) . With this formulation, RTI is successfully simulated [9]. However, the surface tension from this formulation changes its direction across interfaces, which is illustrated via a stationary bubble (density ratio 3, viscosity ratio 1) in Figure 1. The surface tension of this bubble calculated from Equation (18) is plotted along the vertical central cross-section. The surface tension changes its direction across the bubble interface, generating a wiggle over the interface region. This surface tension wiggle can lead to significant velocity and pressure changes over the interface, and always causes numerical instability at large density ratio, although its effect on numerical stability is limited when the density ratio is small.

Although the surface tension in the HCZ model results from intermolecular attraction [9], it is modeled as a body force (see Equation (23)). Thus other continuum surface force formulation can be used to reduce such unphysical oscillations caused by the surface tension. As the LBE multiphase models can be considered as one of diffuse-interface methods [32], Kim's formulation for the diffuse-interface model [26] is used in this work to replace the surface tension formulation in the HCZ model,

$$
F_{\mathrm{S}}=-\kappa \nabla \cdot\left(\frac{\nabla \phi}{|\nabla \phi|}\right)|\nabla \phi| \nabla \phi
$$

where $\kappa$ is the coefficient calculated from Equation (33). The same discretization form of Kim's formulation [26] is adopted for Equation (38). With this new surface tension formulation, the surface tension for the same stationary bubble is shown in Figure 2. No unphysical wiggles in the surface tension profile exist. 

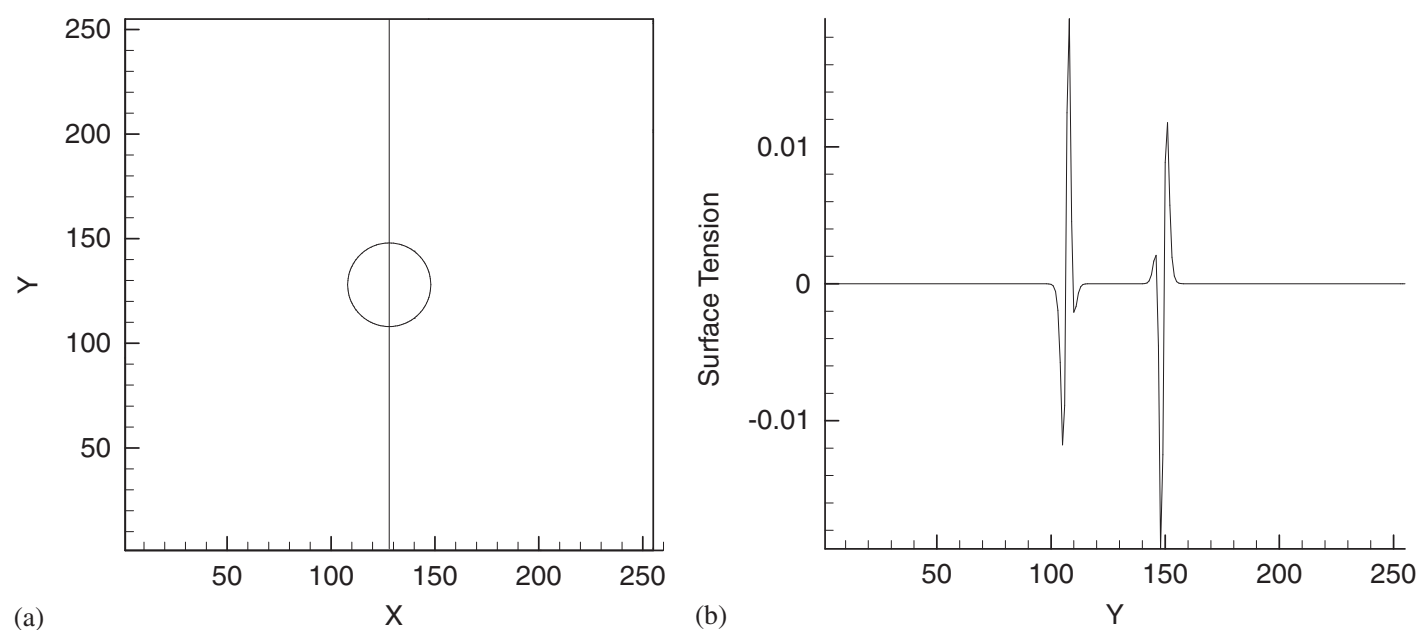

Figure 1. Surface tension profile for a stationary bubble computed from He et al. method.

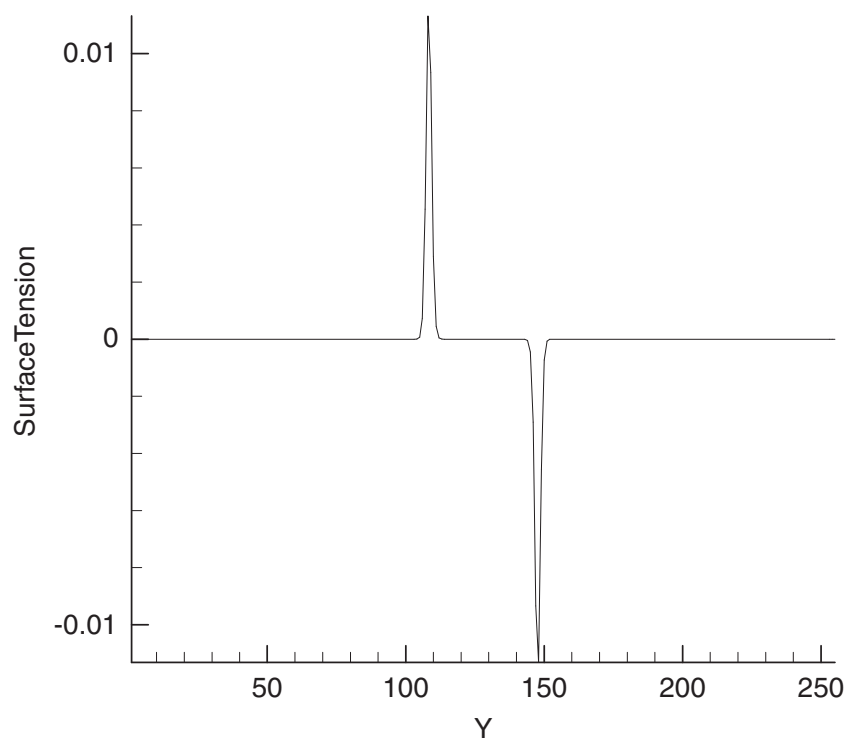

Figure 2. Surface tension calculated by Kim's formulation.

\subsection{A filter technique for index function}

The surface tension calculated from Kim's formulation does not have unphysical wiggle only if monotonic variation of the index function is maintained (see Equation (38)). To ensure the monotonic variation of the index function across the interface, a filter technique used for solving convection-diffusion equation by finite difference [25] is applied to the HCZ model to remove local extremes. The idea of this filter technique is explained as follows.

If the index function has a local extreme with respect to its neighbors, its value would increase (decrease) to the minimum (maximum) value of its neighbors. The filtering algorithm proceeds by first scanning the index function on a node and its neighbors. If it is a local extreme with respect to its neighbors, a correction is made on this node and additional corrections on its neighbors to maintain index function conservation.

Let $\phi_{(I, J)}$ represents the index function on a tested node, $\phi_{(i, j)}$ represents the index function on its eight neighbors, $\phi_{(i, j) \text { min }}$ represent the index function on a neighbor node with minimum 
value, and $\phi_{(i, j) \max }$ represent the index function on a neighbor node with maximum value. Then the filter technique can be described as

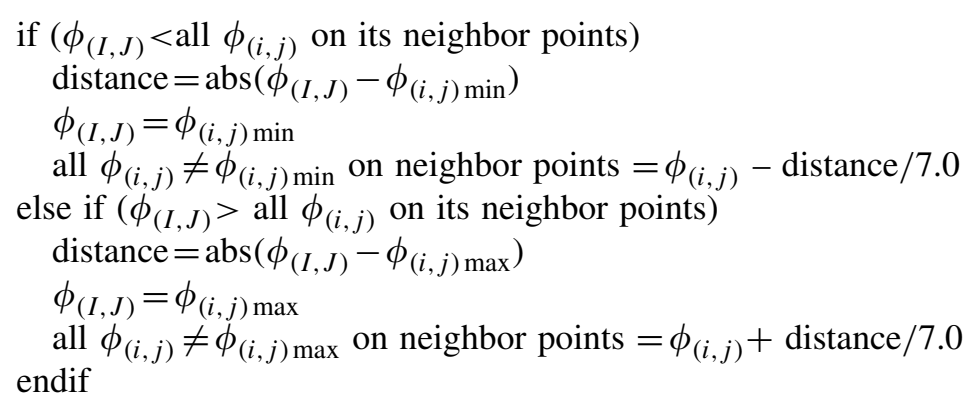

By adjusting the index functions on its neighboring points, this filter algorithm ensures the conservation of the index function. By using this filter local minima/maxima as well as the associated oscillations are removed and monotonic variation of the index function can be maintained. As the filter is only implemented on the nearest and the next nearest neighbor points, the diffusion effect of the filter is limited to only within one lattice. Another advantage of this filter is that it is easy to implement and has much less computational overhead.

\subsection{Mass correction}

For all the LBE multiphase models described in Section 1, the macroscopic volumes of the different phases are calculated from distribution functions. As computational errors, such as large gradient calculations across interfaces, can accumulate and propagate over the whole computational domain, the macroscopic mass may not conserve after long-time computing. This issue of the HCZ model has never been addressed in the literature.

In the HCZ model the density profile is determined by the index function whose evolution is governed by Equation (22). The dispersed phase volume can be obtained from the index function distribution. On the macroscopic level, the index function evolution equation Equation (22) is equivalent to Equation (16). On the RHS of Equation (16) [25], $p(\phi)$ is the thermodynamics pressure in terms of the index function $\phi$, whereas $p(\rho)$ is the hydrodynamic pressure in terms of the density $\rho$. Owing to the presence of interfaces, the RHS of Equation (16) may not be negligibly small in the region near the interface, especially for high density-ratio flows. This term can result in non-conservation of volume/mass that can accumulate over time and destabilize the computation.

In order to ensure the mass conservation, a correction step may be required for high density ratio. In this paper the volume of the dispersed phase, $V$, is corrected using the method described in [33]

$$
\frac{\partial \phi}{\partial \tau}=\left(V-V_{0}\right)|\nabla \phi|
$$

where $V$ is the dispersed phase volume before the correction, $V_{0}$ is the initial volume of the dispersed phase, and $\tau$ is an artificial time. Equation (39) is computed till the steady state $V=V_{0}$ is reached. This correction step is based on the consideration that the density of the fluid in each phase remains the same. If the density changes due to, e.g. pressure variation, then a generalization of the above procedure needs to be devised. Equation (39) can also be recast in another form as

$$
\frac{\partial \phi}{\partial \tau}+\boldsymbol{u} \cdot \nabla \phi=0
$$

where $\boldsymbol{u}=\left(V_{0}-V\right) \nabla \phi /|\nabla \phi|$. This is an advection equation. Thus high-order schemes can be used for this equation to avoid spatial oscillations across discontinuities. In this paper, a finite volume method is used to solve Equation (40) with the second-order essentially non-oscillatory (ENO) scheme for the advection term. In discretizing Equation (40) in space, the lattice node is 
located at the center of a computational cell. The second-order ENO scheme for the advection term discretization is illustrated at the east surface of a computational cell:

$$
\begin{aligned}
& \left.\frac{\partial \phi}{\partial x}\right|_{e 1}=\frac{\phi_{\mathrm{E}}-\phi_{\mathrm{P}}}{\Delta x},\left.\quad \frac{\partial \phi}{\partial x}\right|_{e 2}=\frac{\phi_{\mathrm{P}}-\phi_{\mathrm{W}}}{\Delta x} \quad \text { if } u \geqslant 0 \\
& \left.\frac{\partial \phi}{\partial x}\right|_{e 1}=\frac{\phi_{\mathrm{P}}-\phi_{\mathrm{E}}}{\Delta x},\left.\quad \frac{\partial \phi}{\partial x}\right|_{e 2}=\frac{\phi_{\mathrm{E}}-\phi_{\mathrm{EE}}}{\Delta x} \quad \text { if } u<0 \\
& \left.\frac{\partial \phi}{\partial x}\right|_{e}=\min \left(\operatorname{abs}\left(\left.\frac{\partial \phi}{\partial x}\right|_{e 1},\left.\frac{\partial \phi}{\partial x}\right|_{e 2}\right)\right)
\end{aligned}
$$

where $\mathrm{P}$ is the lattice node and also the center of the finite volume; $\mathrm{E}$ is the east lattice node next to $\mathrm{P}$; $\mathrm{W}$ is the west lattice node next to $\mathrm{P}$. The time derivative is discretized using first-order Euler's scheme.

\section{NUMERICAL ASSESSMENT OF THE PROPOSED TECHNIQUES}

\subsection{Single mode RTI with density ratio 9}

The original HCZ model has been successfully employed to simulate the RTI without surface tension [9]; the comparison with theoretical analysis [34] is very good. At low density ratio, say 3, the jump properties across the interface do not show noticeable numerical instability. In this study, the same RTI problem at a higher density ratio of 9 is used to assess the performance of the extended HCZ model by comparing with the original HCZ model.

The computation is performed in a $2 \mathrm{D}$ rectangular domain of width $W$ with no-slip condition on the top and bottom walls and periodic boundary condition on the sides. The kinematic viscosities of both fluids are set to be the same. The dimensionless parameters, Reynolds number and Atwood number, used to describe the problem, are defined as $R e=\sqrt{W g} W / v$ and $A t=\left(\rho_{\mathrm{h}}-\rho_{1}\right) /\left(\rho_{\mathrm{h}}+\rho_{1}\right)$, where $g$ is gravity, $\rho_{\mathrm{h}}$ and $\rho_{1}$ are the densities of heavy and light fluids, respectively. The time scale in this problem is $T=\sqrt{W / g}$. For an otherwise flat interface, an initial perturbation of $10 \%$ amplitude (normalized by the wavelength) in the interface is specified at $t=0$ to promote the growth of the instability. The more detailed computational setup can be found in [9].

The theoretical growth rate based on linear analysis [34] and numerical growth rate presently obtained are shown in Figure 3. The good agreement between the numerical and theoretical growth rate indicates that the extended HCZ model is capable of capturing accurately the physics of this complex flow $[9,35]$.

Figure 4 shows the evolution of the interface from $10 \%$ initial perturbation computed on a $256 \times 1024$ grid with $R e=2048$ and $A t=0.8$ (density ratio 9). In the early stage $(t / T<1.0$ ), the interface evolution of both the HCZ and the extended HCZ are almost identical. However, at the later stage $(t / T \sim 1.5)$, the HCZ model produces significant oscillations in the near-interface region. After $t / T=1.5$, the numerical instability in the HCZ model causes the simulation to blow up. Comparing with the HCZ model, the extended HCZ model at $t / T=1.5$ performs much better in the interfacial region during the entire period of simulation. The unphysical overshot near the interface of the original $\mathrm{HCZ}$ solution can be clearly seen from the density profile along the central vertical section of the computational domain, as shown in Figure 5, and this overshot grows with time. However, the amplitude of the unphysical overshot of the extended HCZ model is restrained, and does not grow with time. Clearly, the use of the filter in the extended HCZ model has improved the numerical stability. Whether the numerical diffusion introduced by the filter suppresses the physical fluctuations which can trigger turbulence in the later stage is not very clear. Further study is necessary on the characteristics of the filter which can not only suppress unphysical overshot in the density profile, but also capture the physical oscillations. 


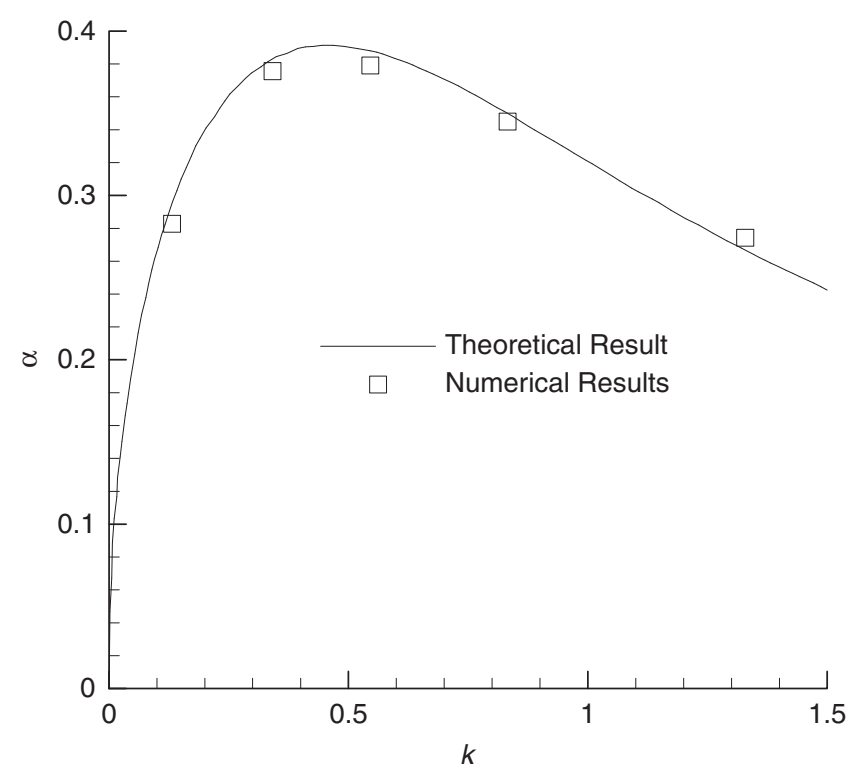

Figure 3. The growth rate $\alpha$ (measured in units of $\left(g^{2} / v\right)^{1 / 3}$ ) of a disturbance vs. its wave numbers $k=2 \pi / W$ (measured in units of $\left(g / v^{2}\right)^{1 / 3}$ ).

To further systematically examine the performance of the present extended HCZ multiphase model, besides the single mode RTI, computations for stationary bubbles, capillary waves, and rising bubbles are carried out in the following sections for larger density ratios up to 100 .

\subsection{Stationary bubble}

3.2.1. A stationary bubble with diameter $d=40$ lattices. The computational parameters for this stationary bubble are listed below:

$$
L a=\frac{\rho_{\mathrm{h}} \sigma d}{\mu_{\mathrm{h}}^{2}}=100, \quad \frac{\rho_{\mathrm{h}}}{\rho_{\mathrm{l}}}=100, \quad \frac{\mu_{\mathrm{h}}}{\mu_{\mathrm{l}}}=10
$$

The computation of this case was performed on a computational domain of $201 \times 201$ lattices. A circular bubble of diameter 40 is placed at the center of the domain with the periodic boundary condition for all boundaries. The surface tension is set to 0.27777 in this case. The dimensionless time is defined as $t_{\mathrm{ch}}=\mu d / \sigma$, which is equal to 480 lattice time steps for this case.

Ideally, the velocity should be zero everywhere and the pressure drop across the interface balances the surface tension force dictated by the Young-Laplace equation:

$$
\Delta p=\frac{\sigma}{R} \text { for } 2 \mathrm{D} \text { bubbles }
$$

The theoretical pressure jump in this case is $\Delta p=\sigma / R=0.1388$. However, due to numerical errors causing imbalance of interfacial stresses, a stationary bubble simulated by continuum surface force methods always has a spurious velocity field. As one of the continuous interface methods, the LBE method is no exception.

Figure 6 shows the pressure profiles of the stationary bubbles with different density ratios, 2, 10, and 100 by using the original HCZ model after only one lattice time. Unphysical wiggle appears at the bubble interface, and its amplitude increases with the density ratio. Figure 7 shows the pressure variation across the bubble computed by the extended HCZ model. The significant unphysical oscillation associated with the original HCZ model is now essentially removed by using the new surface tension formulation with the filter technique.

The density profiles at dimensionless time $t=0$, and $t=100$ (48000 lattice time steps) are shown in Figure 8(a). Very little variation in the density profile is observed. The interface thickness 


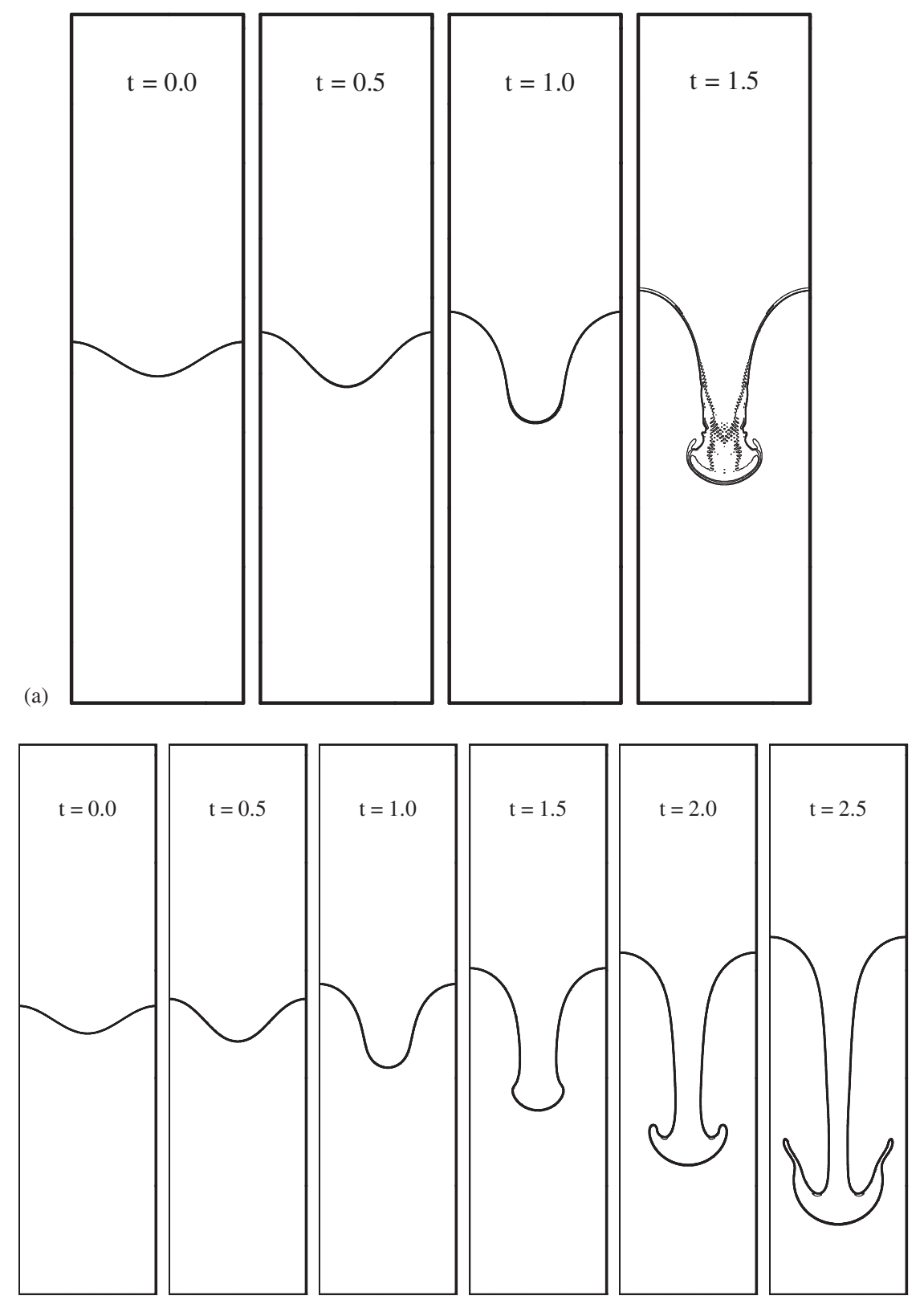

Figure 4. Evolution of the fluid interface from a single mode perturbation for $A t=0.8$ and $R e=2048$. The time is measured in units of $\sqrt{W g}$, where $W$ is the domain width and $g$ is gravity: (a) results from the HCZ model and (b) results from the extended HCZ model.

is maintained within 5-6 lattices, and does not diffuse out with time during the simulation. Furthermore the computed density profiles remain to be monotonic across the interfaces (see Figure 8(b)), which is a significant improvement over the original HCZ model.

3.2.2. Effect of grid resolution on the accuracy of computed pressure jump. There are several ways to characterize computational accuracy of the interfacial problems. In several studies, the dimensionless maximum absolute value of the spurious velocity is often used as an indication of the error in the solution for stationary bubble case [31]. For LBE method, because the lattice speed and lattice spacing are all of unity whereas the resolution of the computation is dictated by the number of lattice across the bubble, it is not possible to maintain the same dimensionless 

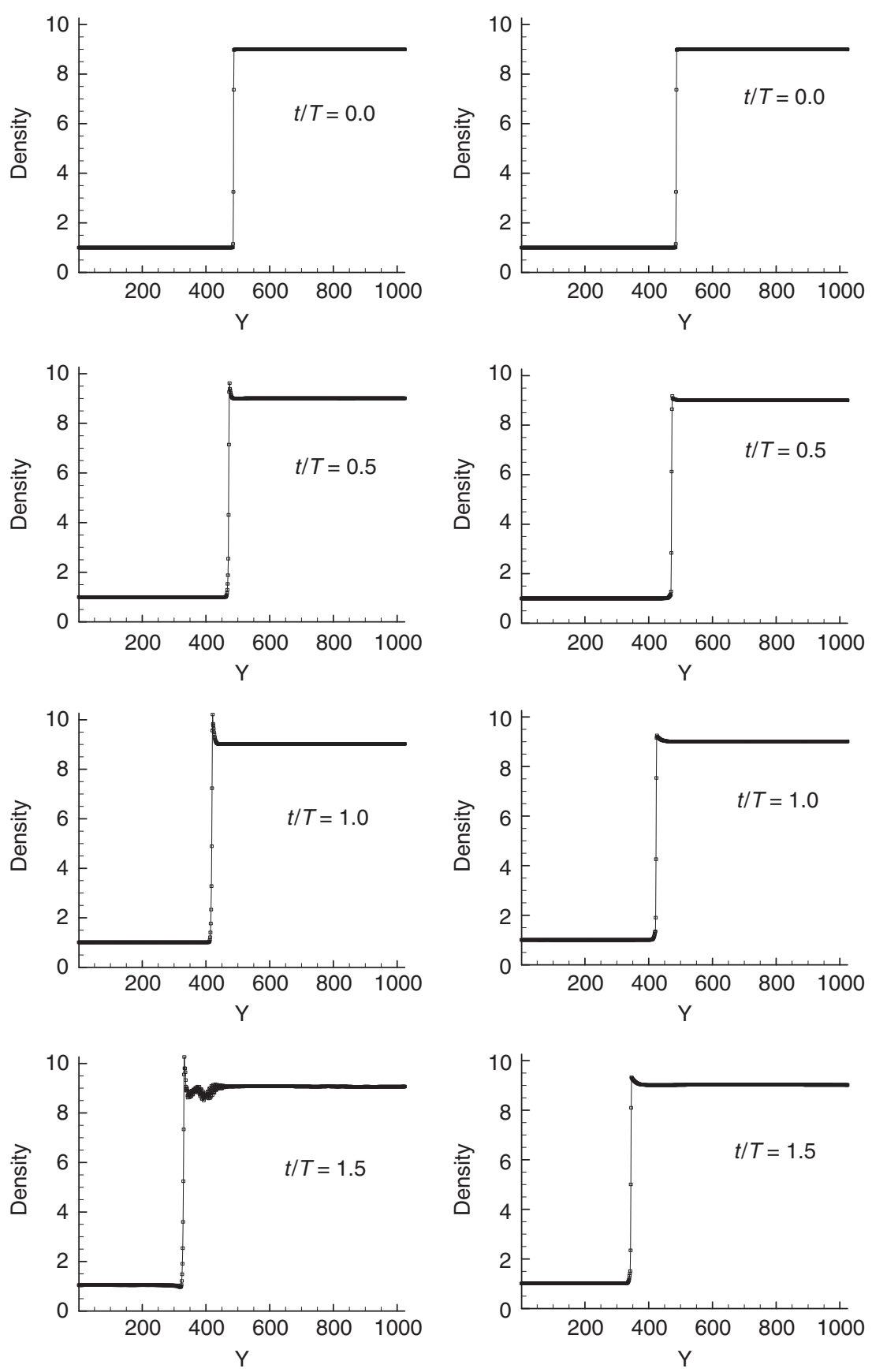

Figure 5. Density profile across the central vertical section of the computational domain at four different time steps. The horizontal axis is the computational grid. The left panel shows the density profiles of the HCZ model and the right panel shows the density profiles of the extended HCZ model $R e=2048$ and $A t=0.8$.

parameters while varying the resolution [36]. Thus an alternative measure for the accuracy of the numerical solution based on the pressure variation is used. Following [37], an average pressure drop is defined as follows:

$$
\Delta p_{\text {num }}=\frac{1}{N_{\mathrm{d}}} \sum_{i, j=1}^{N_{\mathrm{d}}}\left(p_{i, j}-p_{\mathrm{ref}}\right)
$$




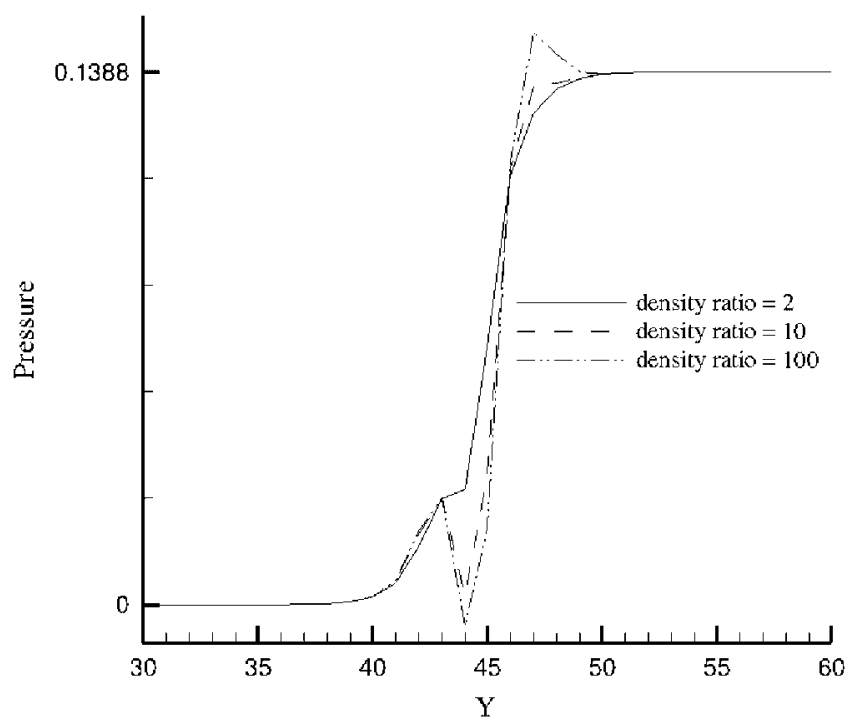

Figure 6. Pressure profiles of stationary bubbles with different density ratios computed by using HCZ model after one lattice time step. The theoretical pressure jumps are 0.1388.

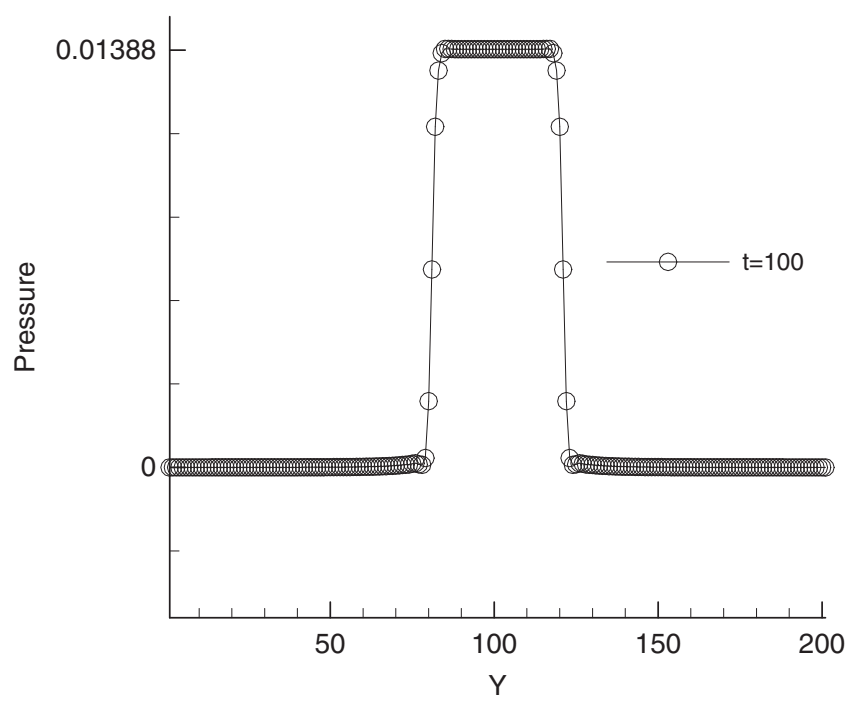

Figure 7. Computational pressure of a stationary bubble computation at $t=100$ for theoretical pressure jump 0.1388. $L a=100$, density ratio 100 , viscosity ratio 10 .

where $N_{\mathrm{d}}$ is the number of lattice nodes lying within the bubble and $p_{\text {ref }}$ is the liquid static pressure far away from the bubble. Because of possible numerical oscillation, the region of the bubble is defined as the region where $\rho \geqslant 1.01 \rho_{\mathrm{g}}$. For a sharp interface, the theoretical value for $\Delta p_{\text {exact }}$ is given by Equation (42). Hence $\left(1-\Delta p_{\text {num }} / \Delta p_{\text {exact }}\right)$ will measure the relative error of the LBE solution in capturing the pressure jump across the interface.

Two stationary bubble cases with different grid resolutions inside the bubbles but with the same Laplace number $(L a=100)$ are computed. Density ratio and viscosity ratio are 100 and 10 , respectively. One bubble has 40 lattices in diameter and the other has 80 lattices. The evolutions of the maximum spurious velocity with dimensionless time are shown in Figure 9. The maximum spurious velocities in both cases approach to constants, indicating that the computational steady states are reached. Table I lists the relative error of the pressure drop, $\left(1-\Delta p_{\text {num }} / \Delta p_{\text {exact }}\right)$, for 

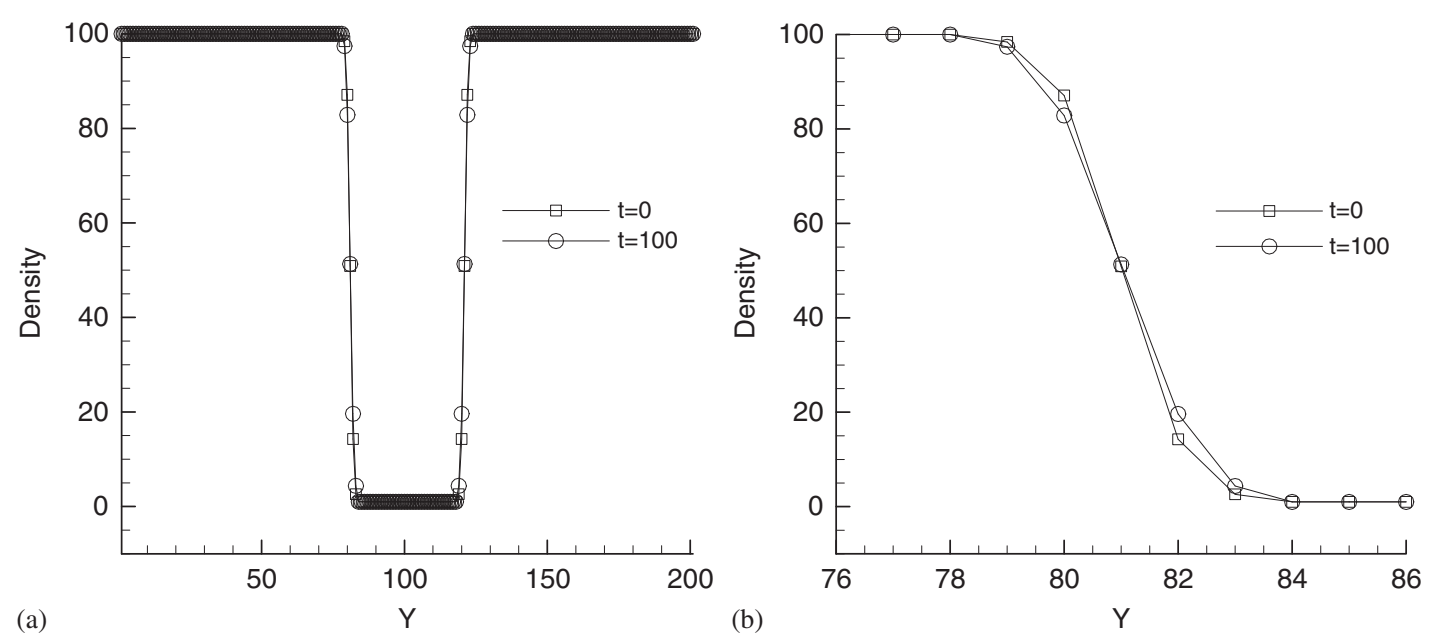

Figure 8. (a) Density profiles of the stationary bubble with diameter $40, L a=100$, density ratio 100 , and viscosity ratio 10 and (b) the zoomed-in density profile at the bubble interface.

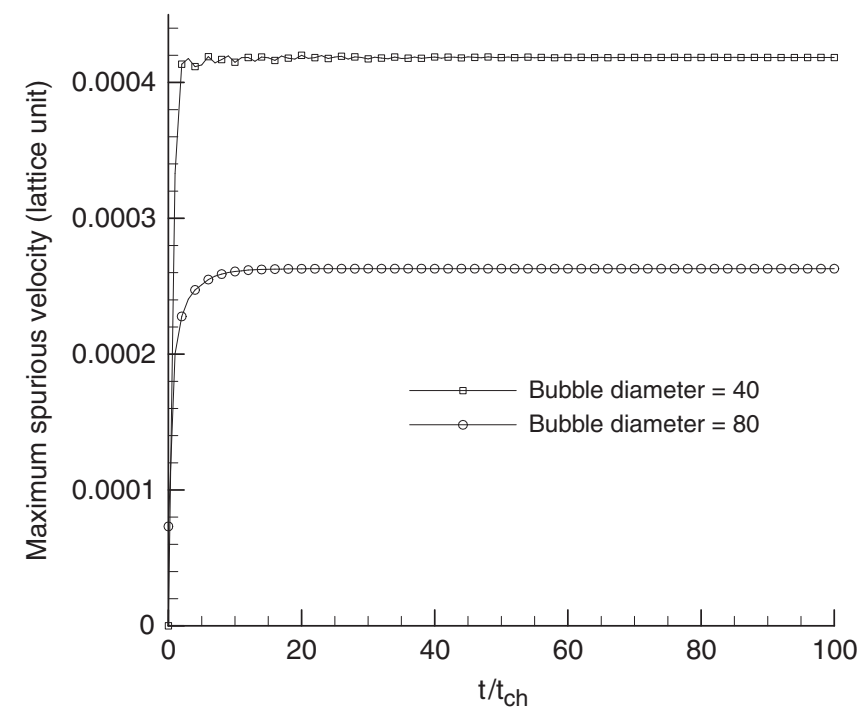

Figure 9. Maximum spurious velocities for these two grid resolutions, $L a=100$, density ratio 100 , viscosity ratio 10 .

Table I. Effect of grid resolution on computed pressure drop for Laplace number 100.

\begin{tabular}{lc}
\hline Grid resolution & $1-\Delta p_{\text {num }} / \Delta p_{\text {exact }}$ \\
\hline 40 lattice in bubble diameter & 0.08 \\
80 lattice in bubble diameter & 0.04 \\
\hline
\end{tabular}

The data were taken after 100 dimensionless time steps and the density and viscosity ratios were set to 100 and 10 , respectively.

these two cases with different grid resolutions. The value for the fine grid case is about half of that for the coarse grid case, indicating that the computation carried out on the fine grid has better accuracy.

The relative errors of the pressure drop of different density ratios are listed in Table II. The Laplace numbers are 100, and viscosity ratios are 10 . The relative errors of the pressure drop in 
Table II. Effect of density ratio on computed pressure drop for Laplace number 100 .

\begin{tabular}{lc}
\hline Density ratio & $1-\Delta p_{\text {num }} / \Delta p_{\text {exact }}$ \\
\hline 3 & 0.05 \\
10 & 0.05 \\
50 & 0.06 \\
100 & 0.08 \\
\hline
\end{tabular}

The data were taken after 100 dimensionless time steps and viscosity ratios was set to 10 .

Table III. Effect of Laplace number on the ratio of numerical pressure drop to theoretical pressure drop: bubble diameter is 40 lattices.

\begin{tabular}{lc}
\hline Laplace number & $1-\Delta p_{\text {num }} / \Delta p_{\text {exact }}$ \\
\hline 100 & 0.08 \\
1000 & 0.08 \\
\hline
\end{tabular}

The data were taken at non-dimensional time $=100$. Density ratio is 100 and dynamics viscosity ratio is 10 .

Table IV. Effect of density ratio on pressure drop: bubble diameter is 40 lattice units.

\begin{tabular}{lc}
\hline Density ratio $=\rho_{1} / \rho_{2}$ & $1-\Delta p_{\text {num }} / \Delta p_{\text {exact }}$ \\
\hline 1 & 0.06 \\
10 & 0.07 \\
100 & 0.08 \\
\hline
\end{tabular}

Viscosity ratio was set to 10 for Laplace number $=100$ and the data were taken at non-dimensional time $=100$.

Table V. Effect of viscosity ratio on pressure drop: bubble diameter is 40 lattice units.

\begin{tabular}{lc}
\hline Viscosity ratio $=\mu_{1} / \mu_{2}$ & $1-\Delta p_{\text {num }} / \Delta p_{\text {exact }}$ \\
\hline 10 & 0.08 \\
100 & 0.07 \\
\hline
\end{tabular}

Laplace number $=100$ and density ratio is 100 . The data were taken at non-dimensional time $=100$.

Table II indicates that the error becomes larger as density ratio increases, although the increase is not significant, which has also been observed by the simulations of the Navier-Stokes solver [31].

The effect of different Laplace numbers on the computational error is also examined. It is found that for $L a=100$ and $L a=1000$ there is no appreciable difference in $\left(1-\Delta p_{\text {num }} / \Delta p_{\text {exact }}\right)$ as shown in Table III. Tables IV and V show the effect of density and viscosity ratios on the solution accuracy. It is observed that they do not cause significant changes in $\left(1-\Delta p_{\text {num }} / \Delta p_{\text {exact }}\right)$. The present finding is similar to that reported in [31] for a stationary bubble by using Navier-Stokes solvers.

\subsection{Capillary wave}

The second test case is a capillary wave, a small-amplitude motion of two superposed viscous fluids with the same viscosity [38]. In this test, gravity is not considered. Initially, the interface between two stationary fluids is set up as a wave with a small amplitude $H_{0}$, as shown in Figure 10 . 


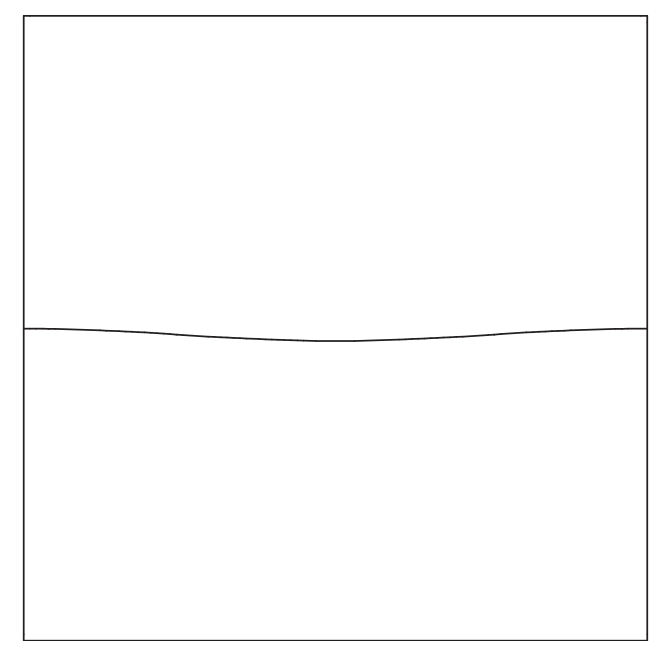

Figure 10. Initial interface profile for a capillary wave simulation, $\omega_{0}=1.13 \times 10^{-3}, \bar{\varepsilon}=0.116$, density ratio 100 .

It damps off with time due to the interaction between viscous force and surface tension. The no-slip boundary condition is used on the top and bottom boundaries and the periodic boundary condition is used on the lateral boundaries. In this problem, the length scale is taken as $k^{-1}$, in which $k$ is the wave number defined as $k=2 \pi / N X$ and $N X$ is the domain width. The time scale is taken as $\omega_{0}^{-1}$, in which $\omega_{0}$ is the frequency defined as $\omega_{0}=\sigma k^{3} /\left(\rho_{1}+\rho_{2}\right)$.

Based on these time and length scales, the dimensionless time and viscosity which characterize the wave motion are

$$
t^{\prime}=\omega_{0} t, \quad \bar{\varepsilon}=\frac{v k^{2}}{\omega_{0}}
$$

where $v$ is the kinematic viscosity of the fluid. With these dimensionless time and viscosity, the theoretic solution for the dimensionless amplitude $a=H / H_{0}$ given by [38] is

$$
a\left(t^{\prime}\right)=\frac{4(1-4 \beta) \bar{\varepsilon}^{2}}{8(1-4 \beta) \bar{\varepsilon}^{2}+1} \operatorname{erfc}\left(\sqrt{\bar{\varepsilon} t^{\prime}}\right)+\sum_{i=1}^{4} \frac{z_{i} \omega_{0}^{2}}{Z_{i}\left(z_{i}^{2}-\bar{\varepsilon} \omega_{0}\right)} \exp \left[\frac{\left(z_{i}^{2}-\bar{\varepsilon} \omega_{0}\right) t^{\prime}}{\omega_{0}}\right] \operatorname{erfc}\left(z_{i} \sqrt{\frac{t^{\prime}}{\omega_{0}}}\right)
$$

where $z_{i}$ are the four complex roots of the following equation

$$
z^{4}-4 \beta \sqrt{\bar{\varepsilon} \omega_{0}} z^{3}+2(1-6 \beta) \bar{\varepsilon} \omega_{0} z^{2}+4(1-3 \beta)\left(\bar{\varepsilon} \omega_{0}\right)^{3 / 2} z+(1-4 \beta)\left(\bar{\varepsilon} \omega_{0}\right)^{2}+\omega_{0}^{2}=0
$$

and $Z_{i}=\prod_{j=1, j \neq i}^{4}\left(z_{j}-z_{i}\right)$. The parameter $\beta$ is given by $\beta=\rho_{1} \rho_{2} /\left(\rho_{1}+\rho_{2}\right)^{2}$.

The test parameters taken here are $\omega_{0}=1.13 \times 10^{-3}, \bar{\varepsilon}=0.116$, and $\rho_{\mathrm{h}} / \rho_{1}$ is 100 . The initial velocity is zero for the whole domain and the distribution functions are assigned to the corresponding equilibrium values. The time evolution of the wave amplitude is shown in Figure 11. The time evolution of the dimensionless amplitude agrees well with that given by the exact solution. The slight difference between them in the early stage may be caused by the numerical initial condition used in implementing the extended HCZ LBE model.

\subsection{Two-dimensional rising bubble}

Two-dimensional single bubbles rising in an initially quiescent fluid due to the buoyancy force are simulated to assess the efficacy of the extended HCZ LBE model in capturing the dynamics of the two-phase flow. In this problem the effects of gravity and the surface tension determine the final 


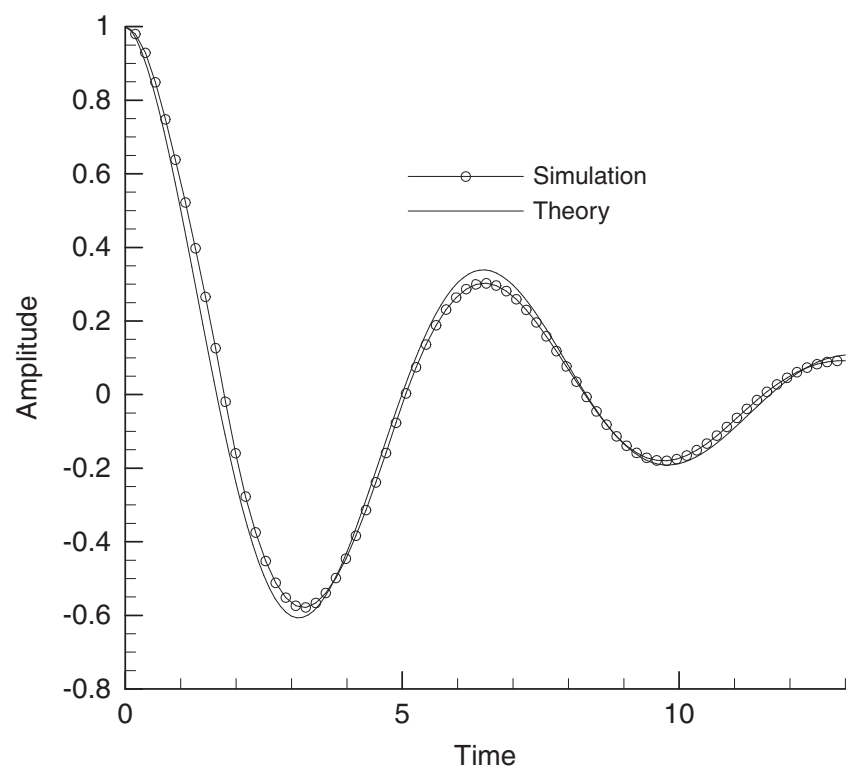

Figure 11. Time evolution of the amplitude of a capillary wave with density ratio 100 .

steady bubble shape. Clift et al. [39] gave a bubble shape diagram in terms of Eötvos number, Morton number, and Reynolds number, defined as

$$
\begin{gathered}
\text { Reynolds number }(R e)=\frac{\rho_{\mathrm{h}} U_{\mathrm{ref}} d}{\mu_{\mathrm{h}}} \\
\text { Morton number }(M)=\frac{g \mu_{\mathrm{h}}^{4}}{\rho_{\mathrm{h}} \sigma^{3}} \\
\text { Eötvos number }(E o)=\frac{\rho_{\mathrm{h}} g d^{2}}{\sigma}
\end{gathered}
$$

The dimensionless time step is given by $t=1 / \sqrt{g / R}$. The simulations carried out here are 2D planar bubbles. The computational domain is a rectangle consisting of $151 \times 801$ lattices. A circular bubble is initially placed at $i=76, j=201$ lattice node with a radius $R=20$ lattices. The initial velocity is set to zero for the whole domain. The no-slip boundary condition is imposed on the top and bottom and the periodic boundary condition is used for the lateral boundaries. The density ratio and viscosity ratio are 100 and 10 , respectively, here.

Three groups of dimensionless parameters are chosen

(1) $E o=0.971, M=1.26 e-3, R e=5.19$

(2) $E o=9.71, M=0.4, R e=6.92$

(3) $E o=97.1, M=100, R e=9.78$

For the first case, the bubble shape should almost be a circle in a 2D simulation. However, without the mass correction, the bubble volume increases with time (Figure 12(a)). At later stages, the bubble shape even changes from a circle to an ellipse due to larger rising velocity. The bubble thus cannot reach its steady state. When the correction step is applied, the bubble volume remains constant and its shape remains almost a circle during the rising process as shown in Figure 12(b).

In this rising bubble simulation, the interfacial compressibility effect is included in the extended HCZ LBE multiphase model. As shown in Equation (23) and discussed in Section 2.1, the interfacial compressibility effect cannot be neglected when density ratio is large. If the last term in Equation (23) is not included, some unphysical mass sources/sinks will be generated along the interface and mass conservation will not be maintained. Figure 13(a) shows the streamlines inside and 


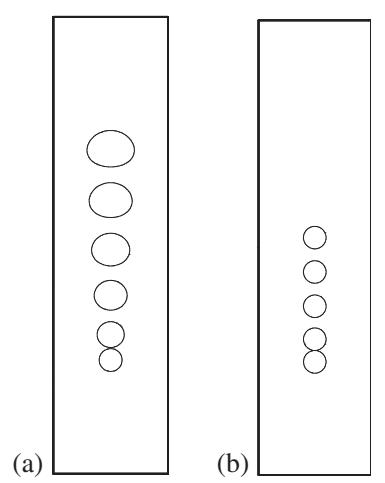

Figure 12 . Rising bubble with $E o=0.971, M o=1.26 e-3$, density ratio $=100$, viscosity ratio $=10$. The time steps in this figure are $t=0,4,8,12,16$, and 20: (a) bubble shapes without mass correction and (b) bubble shapes with mass correction.

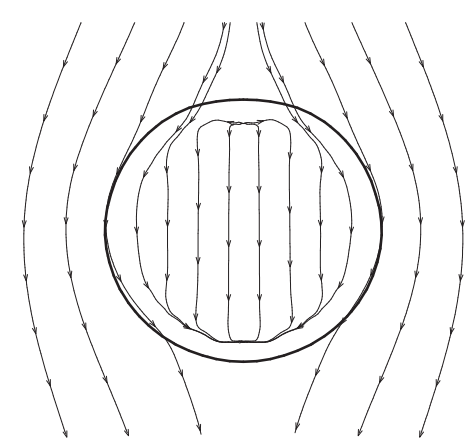

(a)

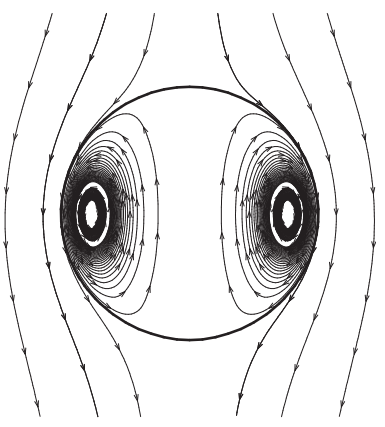

(b)

Figure 13. Streamlines around the bubble at $t=20$ in Figure 12(b): (a) without accounting for the compressibility effect and (b) with accounting for the compressibility effect.

outside this rising bubble without the last term in Equation (23). Figure 13(b) shows the streamlines of the flow inside and outside the rising bubble with the inclusion of the last term in Equation (23). It can be clearly observed that there are mass sources/sinks next to the interface inside the bubble if the interfacial compressibility effect is not included; and these mass sources/sinks can be entirely removed when the interfacial compressibility effect is included. Figure 14 shows the volume and velocity history for this case with mass correction. The volume of this bubble does not change with time and the bubble velocity increases at early time steps and then reaches its steady state value.

Figure 15 shows the absolute surface tension, pressure gradient, and density gradient along the central vertical line of the second case with $E o=9.71, M=0.4, R e=6.92$, density ratio 100 , and viscosity ratio 10 at dimensionless time $t=12$. The surface tension in this figure is comparable to the pressure gradient, indicating that the surface tension is one of the main balancing sources for the pressure gradient around the bubble interface. This figure also shows that the pressure gradient is much smaller than the density gradient around the bubble interface, which is required by Equation (36) in the present model. Similar observations for other cases are also obtained but are not presented for brevity.

Figure 16 shows the computed bubble shapes for all these three cases. Typical bubble shapes are captured using the extended HCZ LBE model. Figure 17 shows the corresponding time evolutions of these bubbles. Owing to larger shape deformation, the dimpled-ellipsoidal bubble rises faster than the other two bubbles. 

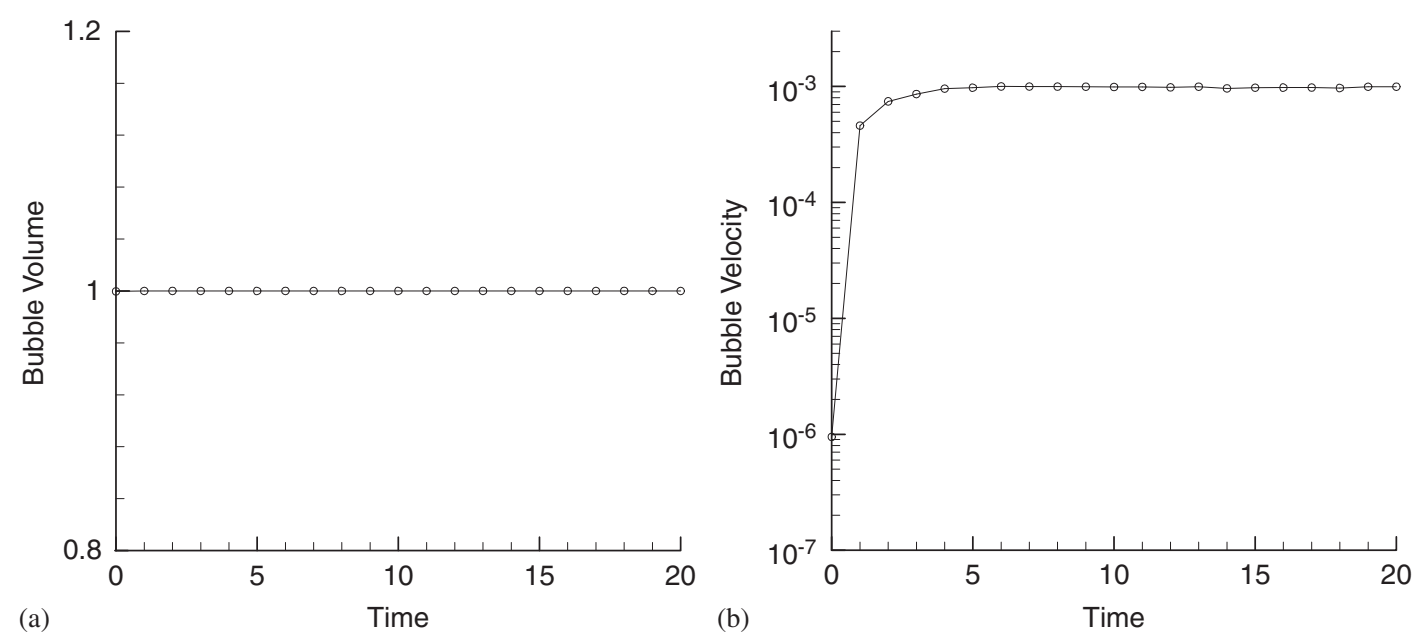

Figure 14. Time evolution of the volume (a) and velocity (b) of the rising bubble with mass correction, as shown in Figure 12(b).

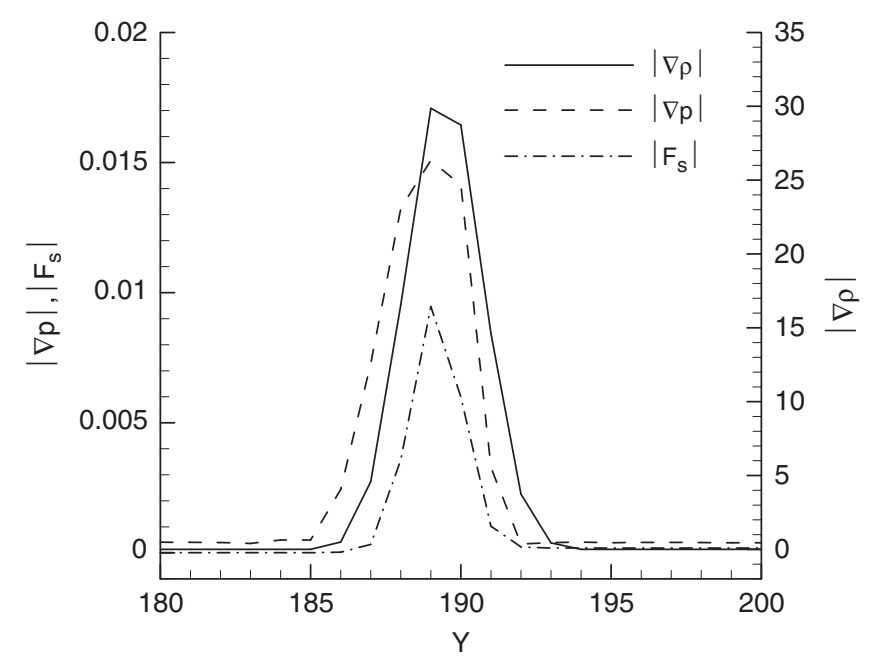

Figure 15. Absolute surface tension, pressure gradient, and density gradient along the central vertical line across the rising bubble with density ratio 100 , viscosity ratio $10, E o=9.71, M=0.4$, $R e=6.92$. Dimensionless time is $t=12$.

Figure 18 shows the velocity histories of these three bubbles. Because of the larger shape deformation compared to cylindrical and ellipsoidal bubbles, the velocity of the dimpled-ellipsoidal bubble exhibits larger oscillations at the early rising stages. At later rising stages, all three bubble velocities do not change significantly, indicating that their steady states are reached.

Figure 19 shows the density profiles of these three bubbles on the central vertical cross-section at $T=0,15$ and 30, respectively. From these density profiles, it can be observed that the interfaces do not diffuse out, and the interface thickness is maintained within five to six grids. Thus the capability of the HCZ model for non-diffused interface thickness is still maintained when the density ratio is up to $O(100)$. This feature of this model is very desirable for interfacial dynamics.

Figure 20 shows the evolution of another rising bubble during a period of six dimensionless time steps with $E o=97.1, M=0.971, R e=31.2$, density ratio 100 , and viscosity ratio 10 . In the 


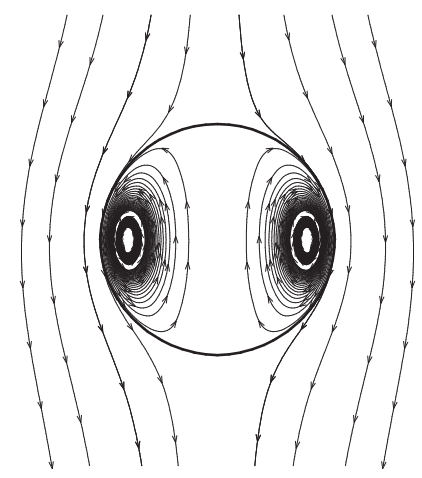

(a)

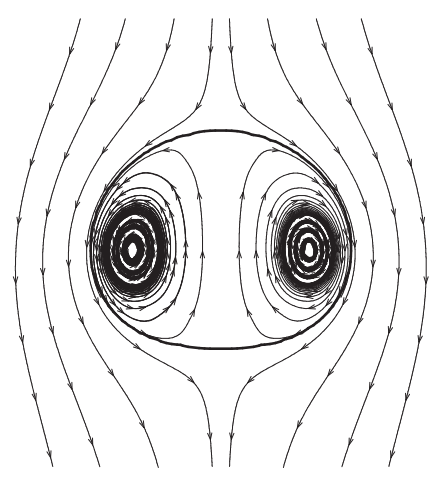

(b)

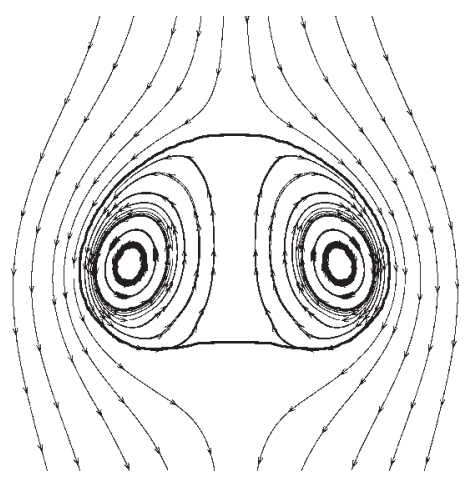

(c)

Figure 16. Computed bubble shapes, density ratio 100, viscosity ratio 10: (a) cylindrical, $E o=0.971, M=1.26 e-3, R e=5.19$; (b) ellipsoidal, $E o=9.71, M=0.4, R e=6.92$; and (c) dimpled-ellipsoidal, $E o=97.1, M=100, R e=9.78$.

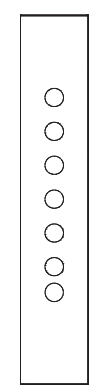

(a)

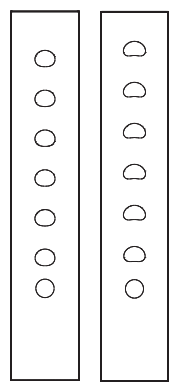

(b) (c)

Figure 17. Time evolutions of rising, density ratio 100 , viscosity ratio 10: (a) cylindrical, $E o=0.971, M=1.26 e-3, R e=5.19$; (b) ellipsoidal, $E o=9.71, M=0.4, R e=6.92$; and (c) dimpled-ellipsoidal, $E o=97.1, M=100, R e=9.78$.

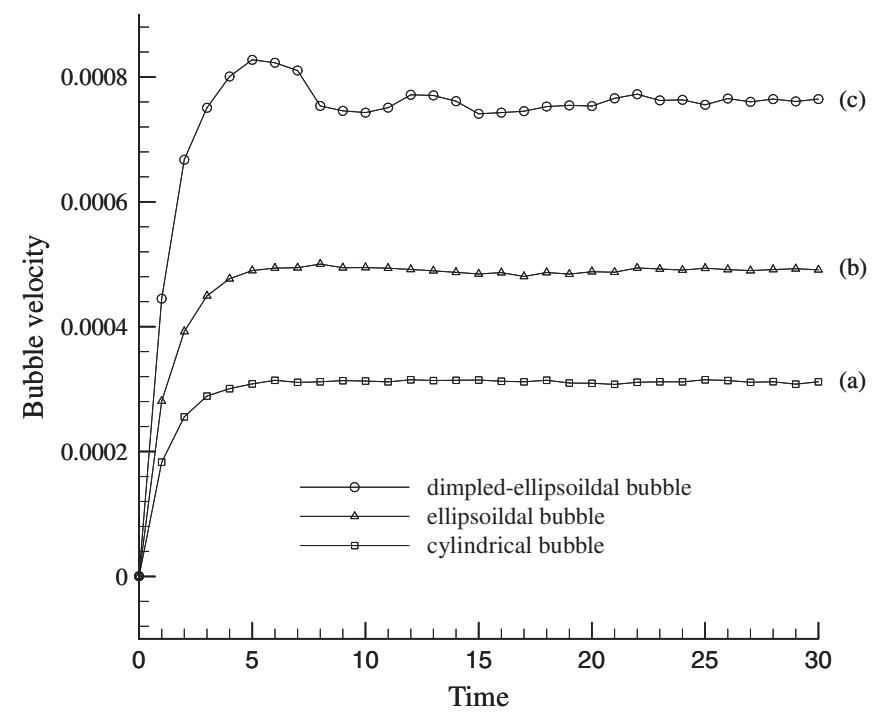

Figure 18. Time evolutions of bubble velocities for density ratio 100 , viscosity ratio 10 : (a) cylindrical, $E o=0.971, M=1.26 e-3, R e=5.19$; (b) ellipsoidal, $E o=9.71, M=0.4$, $R e=6.92$; and (c) dimpled-ellipsoidal, $E o=97.1, M=100, \operatorname{Re}=9.78$. 

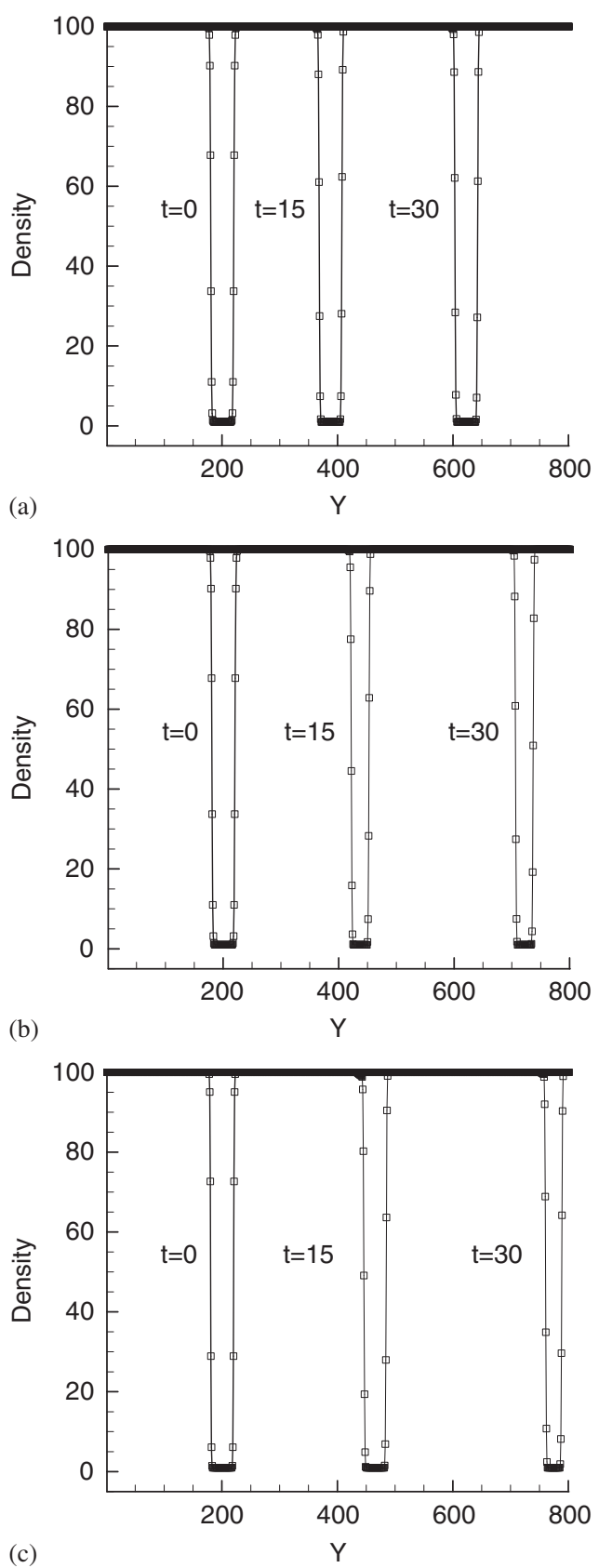

Figure 19. Density profiles of rising bubbles, density ratio 100, viscosity ratio 10: (a) cylindrical, $E o=0.971, M=1.26 e-3, R e=5.19$; (b) ellipsoidal, $E o=9.71, M=0.4, \operatorname{Re}=6.92$; and (c) dimpled-ellipsoidal, $E o=97.1, M=100, \operatorname{Re}=9.78$.

present planar 2D planar simulation, the bubble undergoes complex deformation dynamics and breaks into two larger bubbles with four satellites at the dimensionless time $t=6$.

\section{SUMMARY AND DISCUSSIONS}

In this paper, some issues of the HCZ's LBE model for immiscible multiphase flows are assessed. An extended HCZ model based on a filter technique is developed. A surface tension 


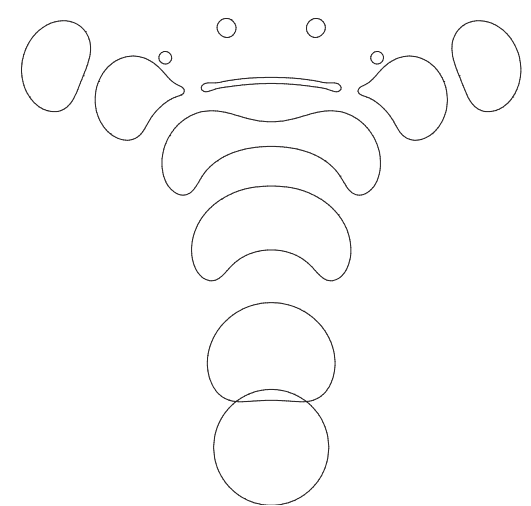

Figure 20. Time evolutions of a rising bubbles, density ratio 100 , viscosity ratio 10 with $E o=97.1, M=0.971$, and $R e=31.2$.

formulation from diffuse-interface methods is adopted to replace that in the HCZ model and to reduce unphysical oscillations in the surface tension across interfaces. The compressibility effect and mass correction are added to the HCZ model to preserve the phase volume and remove unphysical mass sources/sinks. A filtering technique is used to maintain monotonic variation of the index function across interfaces that results in significant reduction of unphysical wiggles in the surface tension profile. The performance of the extended HCZ LBE model is assessed using computations of single mode RTI, stationary bubbles, capillary wave, and rising 2D planar bubbles. The computational results demonstrate that the extended HCZ model can be used to simulate flows with large density ratio up to $O(100)$ while maintaining the interface thickness to within five to six grids through a very long period of time.

The mass conservation issue from the long-time accumulation of the modeling and/or numerical errors in the HCZ model is investigated in this paper. The corresponding mass correction procedure is applied to maintain the mass conservation on the macroscopic level, i.e. correcting the index function via Equation (40). Although this correction yields correct physics, it consumes the most computational time in one lattice time step. This step causes significant reduction in the efficiency of the LBE method. To maintain the efficiency of the LBE method, further efforts should be made to directly correct the mesoscopic distribution functions, rather than the macroscopic index function. However, correcting distribution functions could be much more difficult because, on one lattice node, more distribution functions (for example, nine distribution functions in D2Q9 model) need to be corrected on the mesoscopic level rather than one index function on the macroscopic level.

Further efforts are needed to treat multiphase flows with much higher density ratio of 1000 because as the density ratio becomes higher, the gradient of $\psi(\rho)$ in Equation (23) can still generate numerical instability in pressure and velocity.

\section{APPENDIX A: CONTINUITY EQUATION OF THE EXTENDED HCZ MODEL}

The second LBE in the extended HCZ model is

$$
\begin{aligned}
g_{\alpha}\left(x+e_{\alpha} \Delta t, t+\Delta t\right)-g_{\alpha}(x, t)= & -\frac{g_{\alpha}-g_{\alpha}^{\mathrm{eq}}}{\lambda}+\left(\boldsymbol{e}_{\alpha}-\boldsymbol{u}\right) \cdot\left[\Gamma_{\alpha}(\boldsymbol{u})\left(\boldsymbol{F}_{\mathrm{s}}+\boldsymbol{G}\right)-\left(\Gamma_{\alpha}(\boldsymbol{u})-\Gamma_{\alpha}(\mathbf{0})\right) \nabla \psi(\rho)\right] \\
& -\Gamma_{\alpha}(\mathbf{0}) \frac{\mathrm{d} \psi}{\mathrm{d} \rho} \rho \nabla \cdot \boldsymbol{u}
\end{aligned}
$$

and the corresponding equilibrium distribution function is

$$
g_{\alpha}^{\mathrm{eq}}=\omega_{\alpha}\left[p+\rho R T\left(\frac{3 \boldsymbol{e}_{\alpha} \cdot \boldsymbol{u}}{c^{2}}+\frac{9\left(e_{\alpha} \cdot \boldsymbol{u}\right)^{2}}{2 c^{4}}-\frac{3 \boldsymbol{u}^{2}}{2 c^{2}}\right)\right]
$$


Note that the density in (A2) is not a constant for multiphase flows. Equation (A2) can be recast as

$$
\begin{gathered}
g_{\alpha}^{\mathrm{eq}}=\omega_{\alpha}\left[p-\rho R T+\rho R T\left(1+\frac{3 \boldsymbol{e}_{\alpha} \cdot \boldsymbol{u}}{c^{2}}+\frac{9\left(\boldsymbol{e}_{\alpha} \cdot \boldsymbol{u}\right)^{2}}{2 c^{4}}-\frac{3 \boldsymbol{u}^{2}}{2 c^{2}}\right)\right] \\
g_{\alpha}^{\mathrm{eq}}=\omega_{\alpha}\left[\psi+\rho R T\left(1+\frac{3 \boldsymbol{e}_{\alpha} \cdot \boldsymbol{u}}{c^{2}}+\frac{9\left(\boldsymbol{e}_{\alpha} \cdot \boldsymbol{u}\right)^{2}}{2 c^{4}}-\frac{3 \boldsymbol{u}^{2}}{2 c^{2}}\right)\right]
\end{gathered}
$$

The macroscopic equations can be obtained from Chapmann-Enskog expansion. If Equation (A1) is expanded by Taylor expansion, we can have:

$$
\begin{aligned}
& \Delta t\left[\frac{\partial}{\partial t}+\left(e_{\alpha} \cdot \nabla\right)\right] g_{\alpha}+\frac{(\Delta t)^{2}}{2}\left[\frac{\partial}{\partial t}+\left(e_{\alpha} \cdot \nabla\right)\right]^{2} g_{\alpha}+O\left(\Delta t^{3}\right)=-\frac{g_{\alpha}-g_{\alpha}^{\mathrm{eq}}}{\tau}+\left(\boldsymbol{e}_{\alpha}-\boldsymbol{u}\right) \cdot\left[\Gamma(\boldsymbol{u})\left(\boldsymbol{F}_{\mathrm{s}}+\boldsymbol{G}\right)\right. \\
& \left.-\left(\Gamma_{\alpha}(\boldsymbol{u})-\Gamma_{\alpha}(\mathbf{0})\right) \nabla \psi(\rho)\right] \Delta t \\
& -\Gamma_{\alpha}(\mathbf{0}) \frac{\mathrm{d} \psi}{\mathrm{d} \rho} \rho \nabla \cdot \boldsymbol{u} \Delta t
\end{aligned}
$$

The Chapmann-Enskog expansions are

$$
\begin{aligned}
\frac{\partial}{\partial t} & =\varepsilon \frac{\partial}{\partial t_{1}}+\varepsilon^{2} \frac{\partial}{\partial t_{2}}+\cdots \\
\frac{\partial}{\partial x} & =\varepsilon \frac{\partial}{\partial x_{1}} \\
g_{\alpha} & =g_{\alpha}^{\mathrm{eq}}+\varepsilon g_{\alpha}^{1}+\varepsilon^{2} g_{\alpha}^{2}+\cdots \\
\Gamma_{\alpha}(\boldsymbol{u}) & =\Gamma_{\alpha}^{\mathrm{eq}}(\boldsymbol{u})+\varepsilon \Gamma_{\alpha}^{1}(\boldsymbol{u})+\varepsilon^{2} \Gamma_{\alpha}^{2}(\boldsymbol{u})+\cdots
\end{aligned}
$$

Note that $(\Gamma(\boldsymbol{u})-\Gamma(\mathbf{0})) \nabla \psi(\rho)=\varepsilon(\Gamma(\boldsymbol{u})-\Gamma(\mathbf{0})) \nabla_{1} \psi(\rho)$, and $(\Gamma(\boldsymbol{u})-\Gamma(\mathbf{0})) \sim O(\boldsymbol{u})$ which is proportional to $\varepsilon$ [9], we can then have

$$
(\Gamma(\boldsymbol{u})-\Gamma(\mathbf{0})) \nabla \psi(\rho) \sim \varepsilon^{2}[9] .
$$

Since $\nabla \cdot \boldsymbol{u}=\varepsilon \nabla_{1} \cdot \boldsymbol{u} \sim \varepsilon^{2}[40]$, it leads to

$$
\Gamma_{\alpha}(0) \rho \frac{\mathrm{d} \psi}{\mathrm{d} \rho} \nabla \cdot \boldsymbol{u} \sim \varepsilon^{2} .
$$

Substituting these expansions into Equation (A5), we have

$$
\begin{aligned}
\varepsilon^{0}:\left(e_{\alpha k}-u_{k}\right)\left[\Gamma_{\alpha}(\boldsymbol{u})\left(F_{s k}+G_{k}\right)\right]= & 0 \varepsilon^{1}: \Delta t \frac{\partial g_{\alpha}^{\mathrm{eq}}}{\partial t_{1}}+\Delta t e_{\alpha k} \frac{\partial g_{\alpha}^{\mathrm{eq}}}{\partial x_{1}}=-\frac{1}{\tau} g_{\alpha}^{1}+\left(e_{\alpha k}-u_{k}\right) \Gamma_{\alpha}^{1}(\boldsymbol{u})\left(F_{s k}+G_{k}\right) \Delta t \\
& \varepsilon^{2}: \Delta t \frac{\partial g_{\alpha}^{\mathrm{eq}}}{\partial t_{2}}+\Delta t \frac{\partial g_{\alpha}^{1}}{\partial t_{1}}+\Delta t e_{\alpha k} \frac{\partial g_{\alpha}^{\mathrm{eq}}}{\partial x_{1 k}}-\frac{\Delta t^{2}}{2}\left(\frac{\partial}{\partial t_{1}}+e_{\alpha k} \frac{\partial}{\partial x_{1 k}}\right)^{2} \\
& \times\left(-\frac{1}{\tau} g_{\alpha}^{1}+\left(e_{\alpha k}-u_{k}\right) \Gamma_{\alpha}^{1}(\boldsymbol{u})\left(F_{s k}+G_{k}\right)\right) \\
= & -\frac{1}{\tau} g_{\alpha}^{2}+\left(e_{\alpha k}-u_{k}\right) \cdot\left[\Gamma_{\alpha}^{2}(\boldsymbol{u})\left(F_{s k}+G_{k}\right)\right] \Delta t \\
& -\left(e_{\alpha k}-u_{k}\right) \cdot\left[\left(\Gamma_{\alpha}(\boldsymbol{u})-\Gamma_{\alpha}(\mathbf{0})\right) \frac{\partial \psi}{\partial x_{1 k}}\right] \Delta t-\Gamma_{\alpha}(0) \rho \frac{\mathrm{d} \psi}{\mathrm{d} \rho} \frac{\partial u_{k}}{\partial x_{1 k}} \Delta t \text { (A9) }
\end{aligned}
$$

Summing equations in (A9) over the phase space and using the following constraints:

$$
\sum_{\alpha} g_{\alpha}^{1}=0, \quad \sum_{\alpha} g_{\alpha}^{2}=0, \quad \sum_{\alpha} \Gamma_{\alpha}^{1}=0, \quad \sum_{\alpha} \Gamma_{\alpha}^{2}=0
$$


we can have

$$
\begin{aligned}
\varepsilon^{0}: 0 & =0 \\
\varepsilon^{1}: \frac{\partial p}{\partial t_{1}}+\nabla_{1} \cdot(\rho R T \boldsymbol{u}) & =0 \Rightarrow \frac{\partial \psi}{\partial t_{1}}+\frac{\partial \rho R T}{\partial t_{1}}+\nabla_{1} \cdot(\rho R T \boldsymbol{u})=0 \\
\varepsilon^{2}: \frac{\partial p}{\partial t_{2}} & =-\boldsymbol{u} \cdot \nabla_{1} \psi-\frac{\mathrm{d} \psi}{\mathrm{d} \rho} \rho \nabla_{1} \cdot \boldsymbol{u} \Rightarrow \frac{\partial \psi}{\partial t_{2}}+\frac{\partial \rho R T}{\partial t_{2}}=-\boldsymbol{u} \cdot \nabla_{1} \psi-\frac{\mathrm{d} \psi}{\mathrm{d} \rho} \rho \nabla_{1} \cdot \boldsymbol{u}
\end{aligned}
$$

Combining all the equations in (A10), the continuity equation becomes:

$$
\frac{\mathrm{d} \psi}{\mathrm{d} t}+\frac{\partial(\rho R T)}{\partial t}+\nabla \cdot(\rho R T \boldsymbol{u})=-\frac{\mathrm{d} \psi}{\mathrm{d} \rho} \rho \nabla \cdot \boldsymbol{u}
$$

Since the function of $\psi$ is a function of density (Equation (11) in [9]), we have

$$
\frac{\mathrm{d} \psi}{\mathrm{d} t}=\frac{\mathrm{d} \psi}{\mathrm{d} \rho} \frac{\mathrm{d} \rho}{\mathrm{d} t}=\frac{\mathrm{d} \psi}{\mathrm{d} \rho}\left(\frac{\partial \rho}{\partial t}+u \cdot \nabla \rho\right)=-\frac{\mathrm{d} \psi}{\mathrm{d} \rho} \rho \nabla \cdot u
$$

Then the continuity equation is recovered as

$$
\frac{\partial(\rho R T)}{\partial t}+\nabla \cdot(\rho R T \boldsymbol{u})=0
$$

As $R T$ is a constant in the present model, Equation (A13) becomes

$$
\frac{\partial \rho}{\partial t}+\nabla \cdot(\rho \boldsymbol{u})=0
$$

This is the continuity equation of the extended HCZ model.

The derivation of the momentum equation from the present model is exactly the same as that from the HCZ model. It is not given in this paper for brevity.

\section{ACKNOWLEDGEMENTS}

The present work was supported in part by NASA Constellation University Institutes Program (CUIP), Ms Claudia Meyer program manager. R. Mei acknowledges the support by NASA Glenn Research Center under contract NAG3-2930.

\section{REFERENCES}

1. Shyy W, Udaykumar HS, Rao MM, Smith RW. Computational Fluid Dynamics with Moving Boundaries. Taylor and Francis: Washington, DC, 1996 (revised printing 1997, 1998, 2001).

2. Shyy W. Computational Modeling for Fluid Flow and Interfacial Transport. Elsevier: Amsterdam, The Netherlands, 1994 (revised printing 1997).

3. Scardovelli R, Zaleski S. Direct numerical simulation of free-surface and interfacial flow. Annual Review of Fluid Mechanics 1991; 31:567-603.

4. Tryggvason G, Bunner B, Esmaeeli A, Juric D, Al-Rawahi A, Tauber W, Han J, Nas S, Jan Y-J. A front-tracking method for the computations of multiphase flow. Journal of Computational Physics 2001; 169:708-759.

5. Osher S, Fedkiw RP. Level set methods: an overview and some recent results. Journal of Computational Physics 2001; 169:463-502.

6. Shyy W. Multiphase computations using sharp and continuous interface techniques for micro-gravity applications. Computes Rendus Mecanique 2004; 332:375-386.

7. Singh R, Shyy W. Three-dimensional adaptive Cartesian grid method with conservative interface restructuring and reconstruction. Journal of Computational Physics 2007; 224:150-167.

8. Chen S, Doolen D. Lattice Boltzmann method for fluid flows. Annual Review of Fluid Mechanics 1998; 30:329-364.

9. He X, Chen S, Zhang R. A lattice Boltzmann scheme for incompressible multiphase flow and its application in simulation of Rayleigh-Taylor instability. Journal of Computational Physics 1999; 152:642-663.

10. Shan X, Chen H. Lattice Boltzmann model of simulating flows with multiple phases and components. Physical Review E 1993; 47:1815-1819. 
11. Ye T, Mittal R, Udaykumar HS, Shyy W. An accurate Cartesian grid method for viscous incompressible flows with complex immersed boundaries. Journal of Computational Physics 1999; 156:209-240.

12. Ye T, Shyy W, Chung JC. A fixed-grid, sharp-interface method for bubble dynamics and phase change. Journal of Computational Physics 2001; 174:781-815.

13. Chen S, Diemer K, Doolen GD, Eggert K, Fu C, Gutman S, Travis BJ. Lattice gas automata for flow through porous media. Physica D 1991; 47:72-84.

14. Lee T, Lin C. A stable discretization of the lattice Boltzmann equation for simulation of incompressible two-phase flows at high density ratio. Journal of Computational Physics 2005; 206:16-47.

15. Filippova O, Hänel D. A novel lattice BGK approach for low Mach number combustion. Journal of Computational Physics 2000; 158:139-160.

16. Shan X, Chen H. Simulation of non-ideal gases and liquid-gas phase transitions by the lattice Boltzmann equation. Physical Review E 1994; 49:2941-2948.

17. Swift M, Osborn W, Yeomans J. Lattice Boltzmann simulation of nonideal fluids. Physical Review Letter 1995; 75:830-833.

18. He X, Shan X, Doolen GD. A discrete Boltzmann equation model for non ideal gases. Physical Review E 1998; 57:R13-R16.

19. Sankaranarayanan K, Shan X, Kevrekidis IG, Sundaresan S. Analysis of drag and virtual mass forces in bubbly suspensions using an implicit formulation of the lattice Boltzmann method. Journal of Fluid Mechanics 2002; 452:61-96.

20. Holdych DJ, Georgiadis JG, Buckius RO. Migration of a van der Waals bubble: lattice Boltzmann formulation. Physics of Fluids 2001; 13:817-825.

21. Nourgaliev R, Dinh T, Theofanous T, Joseph D. The lattice Boltzmann method: theoretical interpretation, numerics and implications. International Journal of Multiphase Flow 2003; 29:117-169.

22. Chang Q, Iwan J, Alexander D. Application of the lattice Boltzmann method to two-phase Rayleigh-Benard convection with a deformable interface. Journal of Computational Physics 2006; 212:473-486.

23. Inamuro T, Ogata T, Tajima S, Konishi N. A lattice Boltzmann method for incompressible two-phase flows with large density difference. Journal of Computational Physics 2004; 198:628-644.

24. Zheng HW, Shu C, Chew YT. A lattice Boltzmann model for multiphase flows with large density ratio. Journal of Computational Physics 2006; 218:353-371.

25. Shyy W, Chen M, Mittal R, Udaykumar H. On the suppression of numerical oscillations using a non-linear filter. Journal of Computational Physics 1992; 102:49-62.

26. Kim J. A continuous surface tension force formulation for diffuse-interface models. Journal of Computational Physics 2005; 204:784-804.

27. Carnahan NF, Starling KE. Intermolecular repulsions and the equation of state for fluids. AlChE Journal 1972; 81:1184.

28. Carnahan NF, Starling KE. Equation of state for non-attracting rigid spheres. Journal of Chemical Physics 1969; 51:635.

29. Yang X, James A, Lowengrub J, Zheng X, Cristini V. An adaptive coupled level-set/volume-of-fluid interface capturing method for unstructured triangular grids. Journal of Computational Physics 2006; 217:364-394.

30. Zhang R, He X, Chen S. Interface and surface tension in incompressible lattice Boltzmann multiphase model. Computer Physics Communications 2000; 129:121-130.

31. Singh R. Three-dimensional marker-based multiphase flow computation using adaptive Cartesian grid techniques. Ph.D. Dissertation, University of Florida, 2006.

32. Anderson DM, McFadden GB, Wheeler AA. Diffuse-interface methods in fluid mechanics. Annual Review of Fluid Mechanics 1998; 30:139-165.

33. Son G. A numerical method for bubble motion with phase change. Numerical Heat Transfer, Part B 2001; 39:509-523.

34. Chandrasekhar S. Hydrodynamic and Hydromagnetic Stability. Dover: New York, 1981.

35. Singh RK, Chao J, Popescu M, Tai C-F, Mei R, Shyy W. Multiphase/multi-domain computations using continuum and lattice Boltzmann methods. Journal of Aerospace Engineering 2006; 19:288-295.

36. Chao J. Multiscale computational fluid dynamics and heat transfer with interface. Ph.D. Dissertation, University of Florida, 2006.

37. Brackbill JU, Kothe DB, Zemach C. A continuum method for modeling surface tension. Journal of Computational Physics 1992; 100:335-354.

38. Prosperetti A. Motion of two superposed viscous fluids. Physics of Fluids 1981; 24:1217-1223.

39. Clift R, Grace JR, Weber M. Bubbles Drops and Particles. Academic Press: New York, 1978.

40. Dellar PJ. Incompressible limits of lattice Boltzmann equations using multiple relaxation times. Journal of Computational Physics 2003; 190:351-370. 